\title{
A Lagrangian Trajectory View on Transport and Mixing Processes between the Eye, Eyewall, and Environment Using a High-Resolution Simulation of Hurricane Bonnie (1998)
}

\author{
Thomas A. Cram and John Persing* \\ Department of Atmospheric Science, Colorado State University, Fort Collins, Colorado \\ Michael T. MONTGOMERY \\ Department of Meteorology, Naval Postgraduate School, Monterey, California, and NOAA/Hurricane Research Division, \\ Miami, Florida \\ SCOTT A. BRAUN \\ Laboratory for Atmospheres, NASA Goddard Space Flight Center, Greenbelt, Maryland
}

(Manuscript received 2 December 2005, in final form 13 July 2006)

\begin{abstract}
The transport and mixing characteristics of a large sample of air parcels within a mature and vertically sheared hurricane vortex are examined. Data from a high-resolution (2-km horizontal grid spacing) numerical simulation of real-case Hurricane Bonnie (1998) are used to calculate Lagrangian trajectories of air parcels in various subdomains of the hurricane (namely, the eye, eyewall, and near environment) to study the degree of interaction (transport and mixing) between these subdomains. It is found that 1) there is transport and mixing from the low-level eye to the eyewall that carries air possessing relatively high values of equivalent potential temperature $\left(\theta_{e}\right)$, which can enhance the efficiency of the hurricane heat engine; 2 ) a portion of the low-level inflow of the hurricane bypasses the eyewall to enter the eye, and this air both replaces the mass of the low-level eye and lingers for a sufficient time (order $1 \mathrm{~h}$ ) to acquire enhanced entropy characteristics through interaction with the ocean beneath the eye; 3 ) air in the mid- to upper-level eye is exchanged with the eyewall such that more than half the air of the eye is exchanged in $5 \mathrm{~h}$ in this case of a sheared hurricane; and 4) that one-fifth of the mass in the eyewall at a height of $5 \mathrm{~km}$ has an origin in the mid- to upper-level environment where $\theta_{e}$ is much less than in the eyewall, which ventilates the ensemble average eyewall $\theta_{e}$ by about $1 \mathrm{~K}$. Implications of these findings for the problem of hurricane intensity forecasting are briefly discussed.
\end{abstract}

\section{Introduction}

Vortices exhibit the well-known property that mixing between their cores and near environment is strongly suppressed. Examples are manifold, including vortex rings (Vladimirov and Tarasov 1979), mesoscale ocean

* Current affiliation: Department of Mathematics, Colorado State University, Fort Collins, Colorado.

Corresponding author address: Michael T. Montgomery, Department of Meteorology, Naval Postgraduate School, Monterey, CA 93943.

E-mail: mtmontgo@nps.edu

DOI: $10.1175 / J A S 3921.1$

(C) 2007 American Meteorological Society vortices (Robinson 1982), emergent vortices in quasigeostrophic and two-dimensional turbulence (Carnevale et al. 1991; McWilliams et al. 1994), the wintertime stratospheric vortex (McIntyre 1989), and tropical cyclones (TCs; Willoughby 1998). The details of these flows are quite different, yet the tendency for vortices to act as mixing barriers to environmental fluid is believed central to both their emergence and long lifetimes in complex flows (e.g., McWilliams 1984; Mizuta and Yoden 2001). This vortex robustness can be traced to the quasi-elastic behavior of vortices, which results from having mechanisms that can oppose disturbances that are either axisymmetric [via centrifugal restoration (Howard and Gupta 1962; Charney 1973)] or nonaxisymmetric [via realness of discrete spectra 
(Vladimirov and Tarasov 1980) or via a tendency to axisymmetrize (Melander et al. 1987; Montgomery and Kallenbach 1997; Bassom and Gilbert 1999; Reasor et al. 2004; Schecter and Montgomery 2004)]. If the robustness of a vortex core were to be reduced, however, this could have important consequences on the maintenance of that vortex.

In TCs, the manifestation of some compromise in the robustness of the vortex is the stirring ${ }^{1}$ of air (and attendant properties such as angular momentum or equivalent potential temperature $\theta_{e}$ ) between the various domains of the storm; that is, the eye, the eyewall, and the surrounding environment. Among the key atmospheric mechanisms that have been identified to produce mixing between TC domains are transport by frictionally induced inflow that slips under the eyewall (Persing and Montgomery 2003, hereafter PM03; Braun 2002); stirring by barotropic/baroclinic (inflectional) instabilities that break down the high potential vorticity annulus comprising the eyewall (Schubert et al. 1999; Kossin and Schubert 2001; Nolan and Montgomery 2002; Kwon and Frank 2005), and stirring by an imposed vertical wind shear (Frank and Ritchie 2001; Zehr 2003; Emanuel et al. 2004). Each of these processes has been shown to be capable of significantly altering the intensity of TCs (Wu and Braun 2004; Kossin and Eastin 2001; Bender 1997; Wang 2002; Emanuel 1989, 1997; Montgomery et al. 2006; Frank and Ritchie 2001; Wong and Chan 2004). While signifying a compromise in the robustness of a vortex, these mixing processes can alter the thermodynamics of the hurricane eyewall, whether by enhancement or dilution of relevant thermodynamic properties. Recall for example, that the uptake of moist enthalpy from the underlying ocean and the ascent in the eyewall of the TC represent the isothermal and moist adiabatic legs of an idealized Carnot cycle, respectively. An enhanced intake of energy to the Carnot cycle at this stage suggests an increase in useful work (enhancement of the kinetic energy field) and ultimately an increase in the loss of energy to frictional dissipation at the ocean surface (Emanuel 1986, 1995; PM03).

Evidence in support of the hypothesis that instabilities in the TC eyewall, and their finite amplitude co-

\footnotetext{
${ }^{1}$ We follow the definitions found in Haynes (2002). "Transport" is to carry air with various properties from their source to their sinks. "Stirring" is to bring air with different properties into closer proximity with each other. Stirring that proceeds to the point that the originating characteristics of the air can no longer be distinguished is called "mixing". Mixing will also be used in general discussion where the distinction between these processes is not important.
}

herent structures [such as illustrated in Schubert et al. (1999) and Montgomery et al. (2002)], contribute positively to the intensity of the storm was presented by Emanuel $(1989,1997)$ and PM03. In Emanuel (1997), the breakdown of the vortex sheet comprising the eyewall of a developing storm was shown to be essential for achieving the maximum potential intensity predicted by the Carnot theory (hereafter E-PI). In PM03, eyewall mesovortices bordering the low-level eye and eyewall region were spawned by a (Kelvin-Helmholtz) shear instability on the annulus of high toroidal vorticity associated with the secondary circulation of the hurricane. A characteristic of these coherent structures, not anticipated by Emanuel $(1989,1997)$, was their ability to persistently flux high entropy air from the low-level eye to the eyewall. The temporal eddy and mean transport of high entropy air from the low-level eye to the eyewall was shown to provide supplementary fuel to the heat engine, in addition to what is drawn from the ocean underneath and outside the eyewall. By utilizing this extra fuel, the hurricane can obtain a superintense state (PM03; Montgomery et al. 2006), in which the storm intensity exceeds E-PI. Even if a storm in nature is not superintense, PM03 suggested that the superintensity mechanism may still operate to partially mitigate the negative influences associated with upwelling cooler water, cold downdrafts, environmental vertical shear, or interactions with midtropospheric dry air.

A complementary situation to superintensity is the problem of transport and stirring of midtropospheric dry, low-entropy air from outside the rain area into the eyewall region. This is a form of entrainment that occurs on the vortex scale. Previous work (Simpson and Riehl 1958; Riehl and Malkus 1961; Gray 1968; Emanuel et al. 2004) has suggested that such an exchange process, which can result from an imposed vertical wind shear, can dilute the heat content of the eyewall thereby weakening the intensity of a TC. This process was coined ventilation by Simpson and Riehl (1958). In practical situations, ventilation may occur as vertical wind shear impinges on a storm or, alternatively, as a storm approaches a mid- and upper-tropospheric air mass with low entropy. During the process of producing an intensity forecast, forecasters must decide if the TC core will be adversely affected by this interaction. ${ }^{2}$ In general, ventilation requires dry air to breach the intense vorticity of the eyewall. Idealized modeling studies suggest, however, that this can only occur via nonlinear breakdown of the annulus of high potential vor-

\footnotetext{
${ }^{2}$ In this paper, the core of the $\mathrm{TC}$ refers to the eye and the high-vorticity region of the eyewall.
} 
ticity comprising the eyewall and the attendant stirring between the environment and eyewall air masses (e.g., Schubert et al. 1999). Ventilation is unlike the superintensity mechanism that provides extra fuel from the low-level eye region. Processes that entrain dry air into the TC core will be referred to here as the anti-fuel mechanism.

Little is known quantitatively about the efficiency of the fuel or anti-fuel mechanisms in real-case TCs. In this paper we take the first step in a more systematic study to test and quantify these ideas. The real-case numerical simulation of Hurricane Bonnie (1998) by Braun et al. (2006) employing the nonhydrostatic fifthgeneration Pennsylvania State University-National Center for Atmospheric Research (PSU-NCAR) Mesoscale Model (MM5, version 3.4; Dudhia 1993; Grell et al. 1995) provides a convenient starting point for our examination. By using a large sample of air parcels seeded throughout the storm we build upon the study of Braun (2002) and compute several thousand threedimensional Lagrangian trajectories for air parcels seeded throughout the eye, eyewall, and surrounding regions of Hurricane Bonnie. The large number of Lagrangian trajectories is used to gather a census of the behavior and thermodynamic properties of air parcels in various regions of the simulated hurricane.

Although Bonnie represents only a single case, and results gleaned from it cannot easily be generalized, we believe the methodology developed herein is nonetheless useful for laying the groundwork for a more systematic study of the hurricane mixing phenomena using idealized TC flow configurations. Our objective here is threefold: Identify the nature of the mixing processes that occur within this vertically sheared storm; quantify the efficiency of the mixing between the eye, eyewall, and environment; and relate the current findings on mixing within the vortex to the heat transport that maintains the vortex at approximately constant intensity (in terms of maximum tangential wind) during a period of near-constant vertical shear.

The outline of the paper is as follows. Section 2 describes the model setup and provides an overview of the Hurricane Bonnie simulation. Section 3 describes the Lagrangian trajectory seeding strategy and provides results illustrating the mixing that occurs between various regions of the model hurricane. Figure 1 provides a simplified view of these mixing processes in the context of a hurricane that experiences a moderate degree of vertical wind shear $\left[10 \mathrm{~m} \mathrm{~s}^{-1}(10 \mathrm{~km})^{-1}\right]$. Section 3a focuses on the low-level eye/eyewall interaction, including evidence of the superintensity mechanism (trajectory class I in Fig. 1); section $3 b$ focuses on trajectories in the boundary layer inflow layer and a quantification of the low-level eye mass replacement (class II in Fig. 1 ); section $3 c$ focuses on the upper-level eye/eyewall interaction and investigates the eye containment hypothesis proposed by Willoughby (1998, hereafter W98) (class III in Fig. 1); and section 3d focuses on the interaction of the midlevel environment with the storm and how the environmental vertical shear may serve to ventilate the eyewall and/or eyewall region (class IV in Fig. 1). Section 4 concludes with a summary of the results and objectives for future research.

\section{Model setup and overview of the Bonnie simulation}

Braun et al. (2006) presented an in-depth analysis of a numerical simulation of Hurricane Bonnie using the PSU-NCAR MM5 (version 3.4). We use the same simulation in this study but focus on the mixing characteristics that can be deduced from the use of a large number of Lagrangian trajectories.

\section{a. Experimental design}

Summarizing the numerical setup, four nested grid domains of increasing horizontal resolution were used. Because of computational limits, the simulation was carried out in two steps. First, the two coarser meshes were simulated, using a $36-\mathrm{km}$ horizontal grid spacing and $91 \times 97 \times 27$ grid points in the $x, y$, and $z$ directions for the first grid, and $160 \times 160 \times 27$ grid points at $12-\mathrm{km}$ horizontal grid spacing on the second grid. The simulation on these two outer grid meshes was started at 1200 UTC 22 August 1998 and run for 36 h, with model output saved every hour. A modified version of the Blackadar planetary boundary layer scheme is employed in which surface roughness is calculated for momentum, temperature, and moisture following Garratt (1992) and Pagowski and Moore (2001). Cloud effects are parameterized on the coarse mesh in two ways: the Grell cumulus scheme (Grell et al. 1995) is used to represent unresolved convective processes while the Goddard microphysics scheme (Tao and Simpson 1993; McCumber et al. 1991) is used to represent grid-scale cloud microphysical processes.

A higher-resolution simulation was then carried out using the 1-h output from the 36- and 12-km grid results to provide initial and boundary conditions. The model grids consisted of a $6-\mathrm{km}$ grid with $225 \times 225 \times 27$ grid points and a $2-\mathrm{km}$ nest with $226 \times 226 \times 27$ grid points. The simulation on these high-resolution grids was started $6 \mathrm{~h}$ into the coarse-resolution forecast to allow for model spinup on the $12-\mathrm{km}$ grid, and was carried out for $30 \mathrm{~h}$ until 0000 UTC 24 August. The 2-km grid was moved periodically to keep it centered over the storm. 


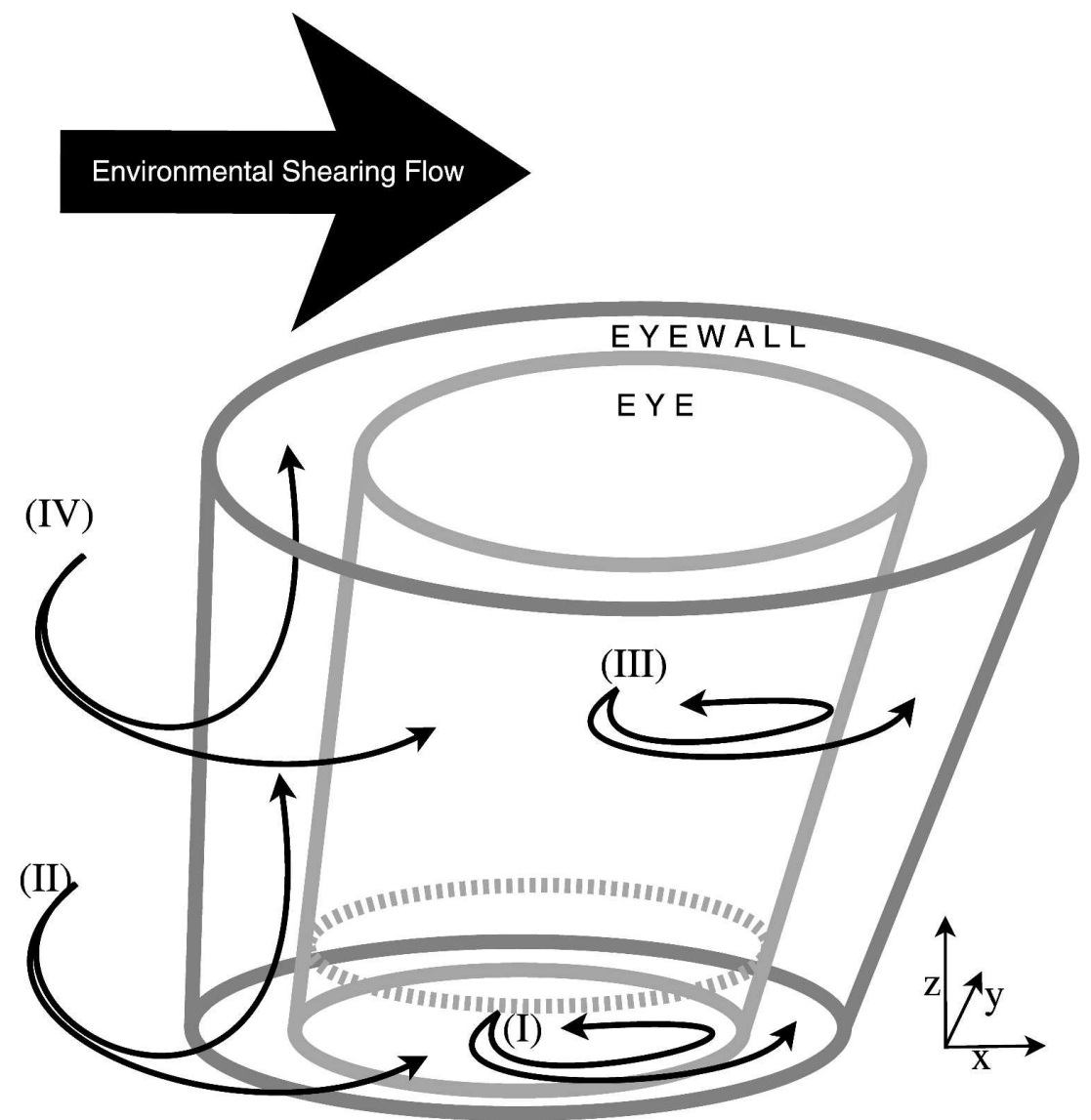

FIG. 1. A simplified schematic of a moderately sheared hurricane. The dark (light) gray lines show the approximate outer (inner) edge of the eyewall. The dotted line represents the inversion level in the eye. The four subsections of section 3 examine different mixing process within the hurricane using different trajectory strategies for the numerical simulation of Hurricane Bonnie by Braun et al. (2006). Section 3a examines the mixing from the low-level eye to the eyewall (trajectory class I) by counting the number of trajectories that enter the eyewall vs those that remain in the eye. Section $3 \mathrm{~b}$ examines the boundary layer inflow from the environment (class II) by considering the properties of trajectories that spend some time in the eye vs those that are transported immediately into the eyewall. Section $3 c$ examines the persistence of air in the mid- and upper-tropospheric eye (class III) by counting the number of trajectories that stir into the eyewall vs those that remain in the eye. Section $3 \mathrm{~d}$ examines the ability of mid- and upper-tropospheric environmental air to stir into the eye or eyewall (class IV).

Model physics were similar to the coarse-grid runs except that no cumulus scheme was used. Further details of the model setup, initialization, and model physics are provided in Braun et al. (2006). For this paper, $t=0 \mathrm{~h}$ will be considered the start of the fine mesh simulation; that is, 1800 UTC 22 August 1998.

This study focuses on a 5-h period from $t=15-20 \mathrm{~h}$, 0900 to 1400 UTC 23 August 1998. The simulated storm during this time undergoes an intensifying trend (Fig. 2). The minimum central surface pressure $p_{\text {sfc }}$ decreases steadily from $961 \mathrm{mb}$ at $14 \mathrm{~h}$ to $952 \mathrm{mb}$ at $20 \mathrm{~h}$. Maxima of tangential wind $v_{\mathrm{sfc}}$ at $z=40 \mathrm{~m}$ during this time vary between 50 and $55 \mathrm{~m} \mathrm{~s}^{-1}$, although there is a slight increasing trend in the 6-h period. The maximum azimuthally averaged tangential wind $\bar{v}_{\text {sfc }}$ increases slightly from approximately 40 to $44 \mathrm{~m} \mathrm{~s}^{-1}$. Comparison with National Hurricane Center (NHC) best track for Hurricane Bonnie at 0600,1200, and 1800 UTC shows $p_{\text {sfc }}$ intensifying $(960,958,955 \mathrm{mb})$ and wind speeds increasing $\left(49,51,51 \mathrm{~m} \mathrm{~s}^{-1}\right)$.

\section{b. Summary of simulated structure}

A complete summary of the simulated structure of the Bonnie simulation can be found in Braun et al. (2006). Several features of particular interest to the current study, however, are summarized below. 


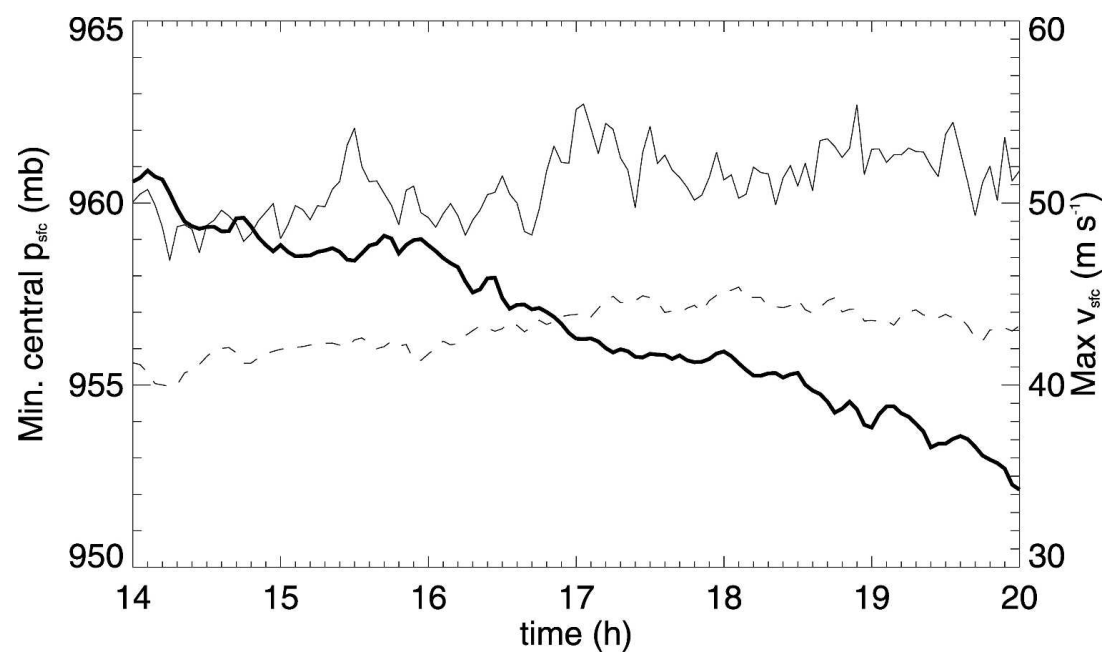

FIG. 2. Time series of minimum central surface pressure (thick line), maximum tangential wind (thin line), and maximum azimuthally averaged tangential wind (dotted line) at the lowest model level $(z=40 \mathrm{~m})$ for the period within the Hurricane Bonnie (1998) simulation of Braun et al. (2006) used here. The period covered here corresponds to 0800-1400 UTC 23 Aug 1998.

The system-scale structure of the storm is primarily influenced by northwesterly environmental deep layer (850-200 mb) vertical shear. Because vertical wind shear has been identified (DeMaria and Kaplan 1994) as a leading predictor of storm intensity and is statistically associated with weaker storms, we anticipate that shear will be an important factor in determining simulated intensity. Simulated radar reflectivity (shown in Fig. 6 in Braun et al. 2006) exhibits an asymmetric storm structure consistent with the influence of northwesterly vertical wind shear. The highest reflectivities appear southeast, east, and north of the storm center, which is downshear and left of the 850-200-mb vertical shear vector calculated about the storm center. The vertical wind shear was calculated inside a radius of 300 $\mathrm{km}$ from the storm center on the 6-km horizontal grid and has a magnitude of approximately $12 \mathrm{~m} \mathrm{~s}^{-1}$ between $z=1$ and $z=12 \mathrm{~km}$; observations from other TCs have shown that at this magnitude, vertical shear effects dominate subsequent storm evolution (e.g., Black et al. 2002). The boundary layer is characterized by inflow (Fig. 3a) in all quadrants outside the radius of maximum wind (RMW), but is stronger southeast, east, and north of the vortex center. ${ }^{3}$ Generally, the peak near-surface inflow is confined below $z=1 \mathrm{~km}$, decreasing to $z=750 \mathrm{~m}$ near the RMW. In all regions of

\footnotetext{
${ }^{3}$ The method for computing the storm center is described in appendix A.
}

the low-level eye except north of the center, radial outflow prevails and reaches magnitudes in excess of $5 \mathrm{~m} \mathrm{~s}^{-1}$. Radial flow into and out of the core of the storm can be found at other levels correlated with the expected wave-1 pattern associated with the environmental mean flow profile that contributes strongly to the vertical wind shear.

Just above the surface inflow layer $(z=1.1 \mathrm{~km})$, the region outside the RMW exhibits radial outflow to the west and inflow to the east of the storm center. Inside the RMW, radial outflow exists directly downshear and left of the deep layer mean vertical shear vector. The outflow inside the RMW at these two levels $(z=453 \mathrm{~m}$ and $1.1 \mathrm{~km}$ ) is associated with the convective updrafts which preferentially form in the downshear side of the eyewall, as described in Braun et al. (2006).

In the middle and upper troposphere $(z=5.6$ and 9.9 $\mathrm{km}$ ) radial outflow occurs south, east, and northeast of the storm center. Inside the RMW, the outflow is particularly strong $\left(u>10 \mathrm{~m} \mathrm{~s}^{-1}\right)$ directly south of the storm center. This region was shown in Braun et al. (2006) to be a favorable area for the initiation of eyewall convective hot towers. While the updrafts presumably form at low levels (below $2 \mathrm{~km}$ ), Braun et al. (2006) noted that these eyewall updrafts extended through the depth of the troposphere shortly after their initiation.

The azimuthal mean storm structure (Fig. 4) generally exhibits an outward sloping eyewall with maximum mean tangential wind $\bar{v}$ of $55 \mathrm{~m} \mathrm{~s}^{-1}$ and mean vertical 
(a)

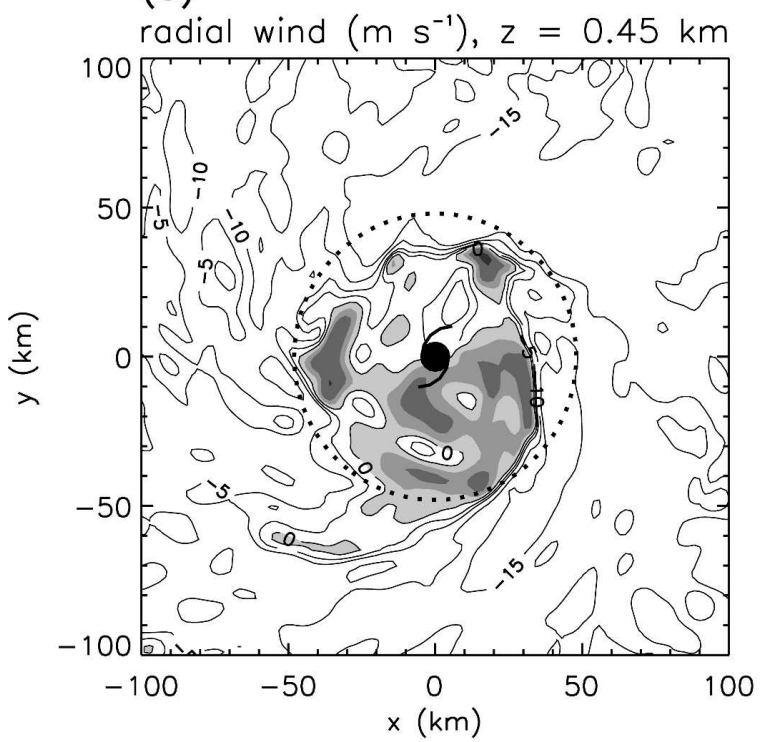

(c)

radial wind $\left(\mathrm{m} \mathrm{s}^{-1}\right), \mathrm{z}=5.58 \mathrm{~km}$

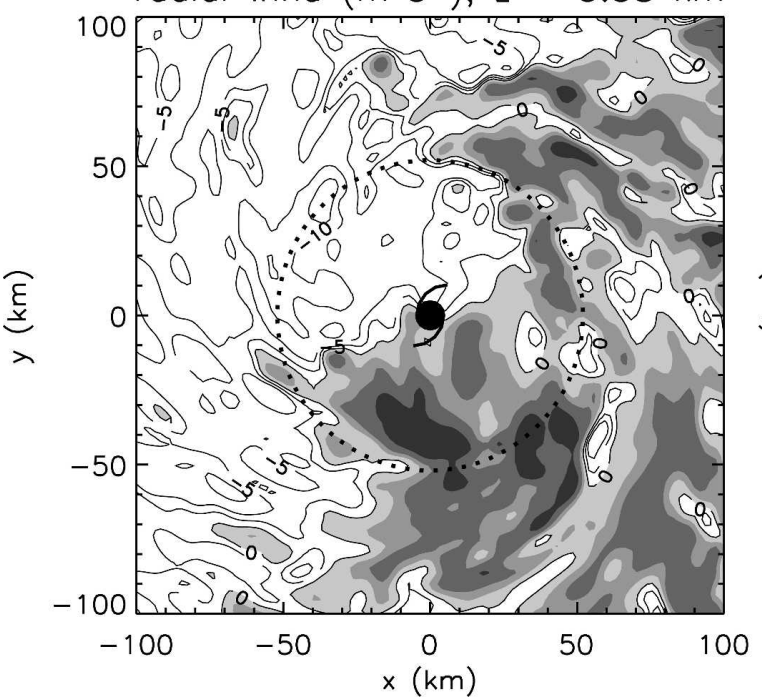

(b)

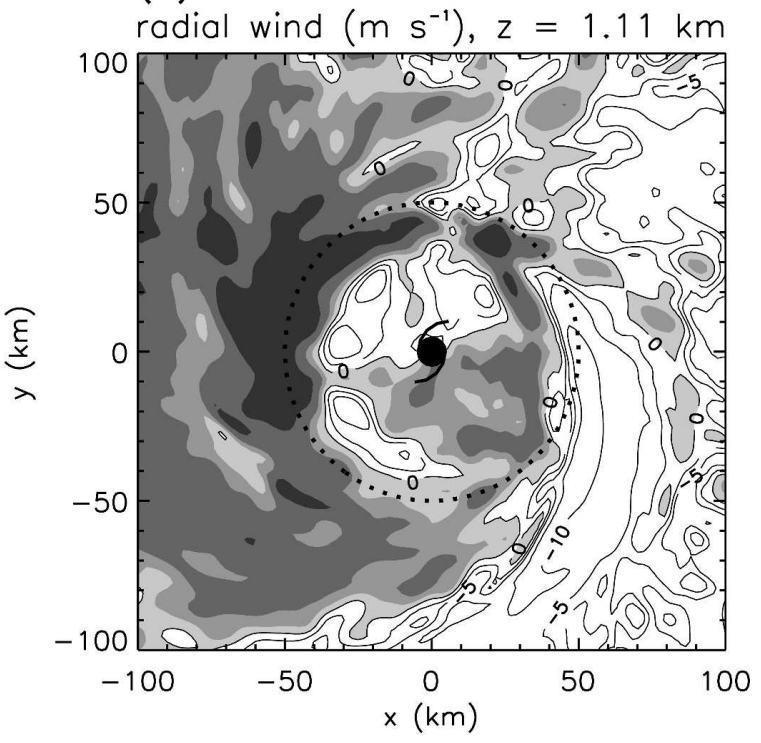

(d)

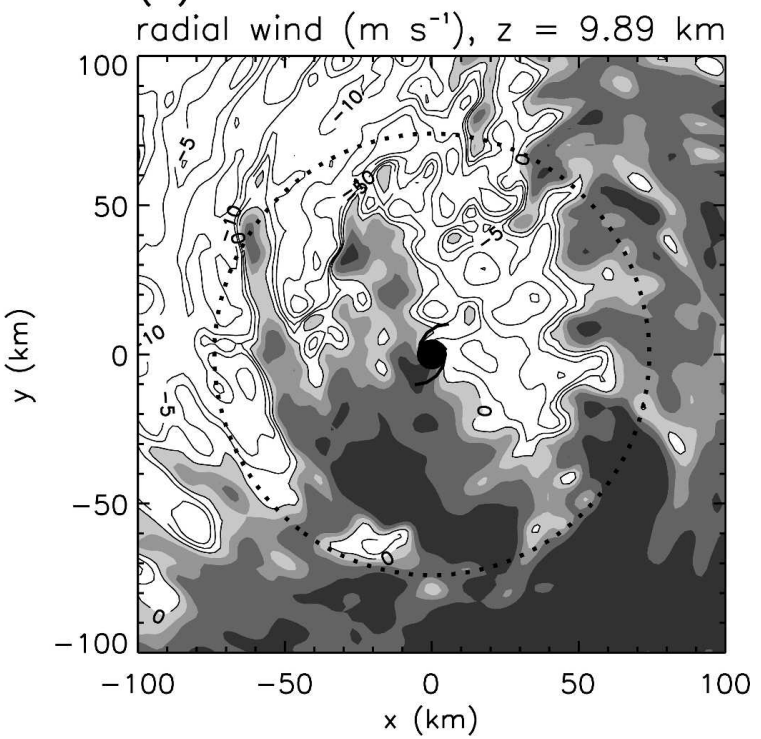

FIG. 3. Contours of radial wind at $z=$ (a) $453 \mathrm{~m}$, (b) $1.1 \mathrm{~km}$, (c) $5.6 \mathrm{~km}$, and (d) $9.9 \mathrm{~km}$ at $t=15 \mathrm{~h}$. Positive values are shaded at levels of $0,2.5,5$, and $10 \mathrm{~m} \mathrm{~s}^{-1}$. Negative values are contoured at $-15,-10,-5$, and $-2.5 \mathrm{~m} \mathrm{~s}^{-1}$. The dotted line represents the radius of maximum mean tangential wind at each level.

velocity $\bar{w}$ of $1.2 \mathrm{~m} \mathrm{~s}^{-1}$. The RMW near the surface at this time $(t=15 \mathrm{~h})$ is $44 \mathrm{~km}$ and gradually moves inward with time to $38 \mathrm{~km}$ at $t=20 \mathrm{~h}$ (not shown). Maximum $\bar{w}$ increases from 1.2 to nearly $2.3 \mathrm{~m} \mathrm{~s}^{-1}$ through the period $t=15-20 \mathrm{~h}$.

The distribution of $\bar{\theta}_{e}$ air (Fig. 4c, following Bolton 1980) suggests a reservoir of high $\theta_{e}$ in the boundary layer of the eye that is characteristic of mature hurricanes (W98; Zhang et al. 2002). In the eye, the maximum in $\bar{\theta}_{e}$ is greater than $376 \mathrm{~K}$ near the surface and reaches a minimum at $z=4 \mathrm{~km}$.

\section{Use of trajectories to study transport, stirring, and mixing processes within the vortex}

Each of the following sections will illustrate the interaction between various regions of the model hurricane using distinct sampling strategies for the initial locations of trajectories. The strategies are detailed separately in each section. A simplified view of a moderately sheared hurricane is shown in Fig. 1 and also describes the transport processes that we are interested in analyzing in this study. The calculation of trajectories 

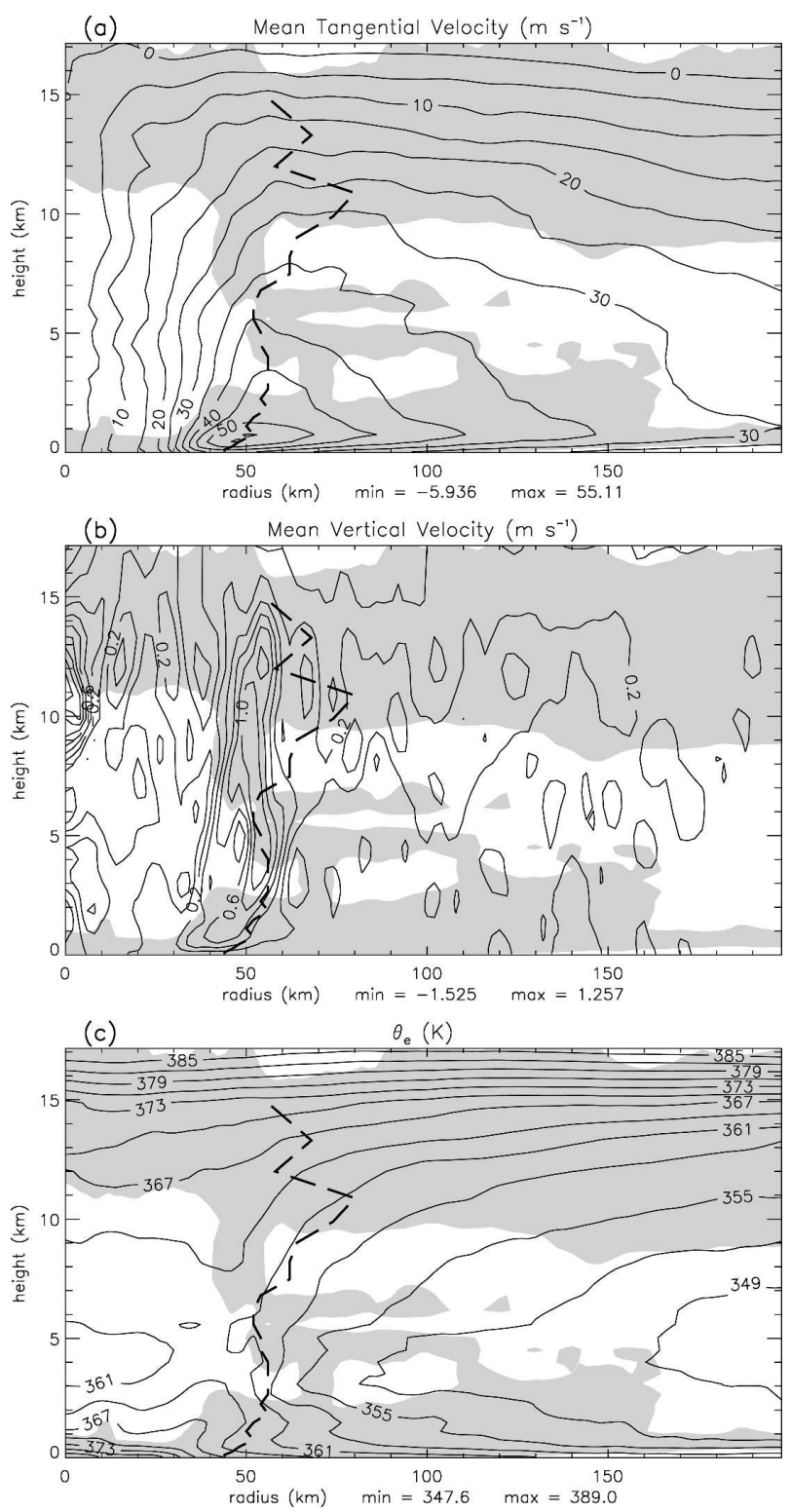

FIG. 4. Azimuthal averages of (a) tangential wind, (b) vertical velocity, and (c) equivalent potential temperature at $t=15 \mathrm{~h}$ in the Hurricane Bonnie simulation by Braun et al. (2006). Gray shading denotes azimuthally averaged relative humidity greater than or equal to $90 \%$. The dashed line is the radius of maximum azimuthally averaged tangential wind as a function of height.

is performed by postprocessing the model output data, which has a temporal output increment of three minutes. Trajectory positions are computed every $10 \mathrm{~s}$ by the technique described in appendix B.

\section{a. The low-level eye-eyewall interaction}

\section{1) The trajectory sample}

In this section, we examine the interaction between the low level eye and the eyewall using forward trajec- tories (class I from Fig. 1) seeded in the moist air of the low-level eye (below $z=1.1 \mathrm{~km}$ ). The trajectories are seeded at $z=453 \mathrm{~m}$ in the eye at $t=900 \mathrm{~min}=15 \mathrm{~h}$ from the start of the inner-grid simulation and are calculated forward for $5 \mathrm{~h}$ from this time. The starting locations are on a rectilinear grid with a $2-\mathrm{km}$ spacing in both $x$ and $y$, matching the specification of the model grid.

The eye is distinguished from the eyewall using the following axisymmetric criteria. The eyewall is defined here as the region in the vicinity of the RMW with azimuthally averaged vertical velocity $\bar{w} \geq 0.25 \mathrm{~m} \mathrm{~s}^{-1}$ and axisymmetric mean relative humidity $\overline{\mathcal{H}} \geq 90 \%$. The low-level eye (below $z=1 \mathrm{~km}$ ) is defined as the region inside the RMW and near the storm center with $\bar{\theta}_{e} \geq 370 \mathrm{~K}$. The eye at $z=1.1 \mathrm{~km}$ is defined as the region inside the RMW and near the storm center with $\bar{w}<0.25 \mathrm{~m} \mathrm{~s}^{-1}$. The eye at mid- to upper levels $(z=5.5$ $\mathrm{km}$ and above) is defined as the region inside the RMW and near the storm center with $\bar{w}<0.25 \mathrm{~m} \mathrm{~s}^{-1}$ and $\overline{\mathcal{H}}<$ $90 \%$. These definitions using axisymmetric properties help distinguish the eyewall from short-lived cumuli in the eye itself. Although it would be preferable to apply the same definition for the eye at $z=1.1 \mathrm{~km}$ as is used at mid- to upper levels, the relatively higher values of $\overline{\mathcal{H}}$ at $1.1 \mathrm{~km}(\sim 90 \%-92 \%)$ do not provide a clear distinction between the mean eye and eyewall and thus precludes the use of a humidity discriminant at this level. The shear-induced asymmetry in the storm lowers the axisymmetric relative humidity below saturation, hence the value of $\overline{\mathcal{H}}=90 \%$ is chosen as the humidity discriminant for defining the mean eyewall (cf. Fig. 4).

\section{2) Transport From the LOW-LEVEL Eye to THE EYEWALL}

A small sample of the trajectories initiated in the eye that enter the intense eyewall updraft are shown in Fig. 5. After first encountering the eyewall, these seven trajectories encounter a downdraft at $z \sim 1-2 \mathrm{~km}$ and approach the center of the storm before becoming entrained into the eyewall updraft. At upper levels $(z \geq 7$ $\mathrm{km}$ ), six of these trajectories detrain into the eye. Vertical velocities of the eyewall updraft encountered by these trajectories range from $1-2 \mathrm{~m} \mathrm{~s}^{-1}$ below $z=3 \mathrm{~km}$ to as much as $10 \mathrm{~m} \mathrm{~s}^{-1}$ above this height (not shown). The initial $\theta_{e}$ associated with the trajectories is between 372 and $376 \mathrm{~K}$, confirmation that these trajectories are originating from the reservoir of relatively high $\theta_{e}$ located in the low-level eye $\left(\theta_{e}\right.$ greater than $370 \mathrm{~K}$ extend up to $z=750 \mathrm{~m}$ at the storm center; cf. Fig. 4c). As the trajectories rise in the eyewall updraft, the associated $\theta_{e}$ decreases approximately $5 \mathrm{~K}$. The vertical profile of $\theta_{e}$ 

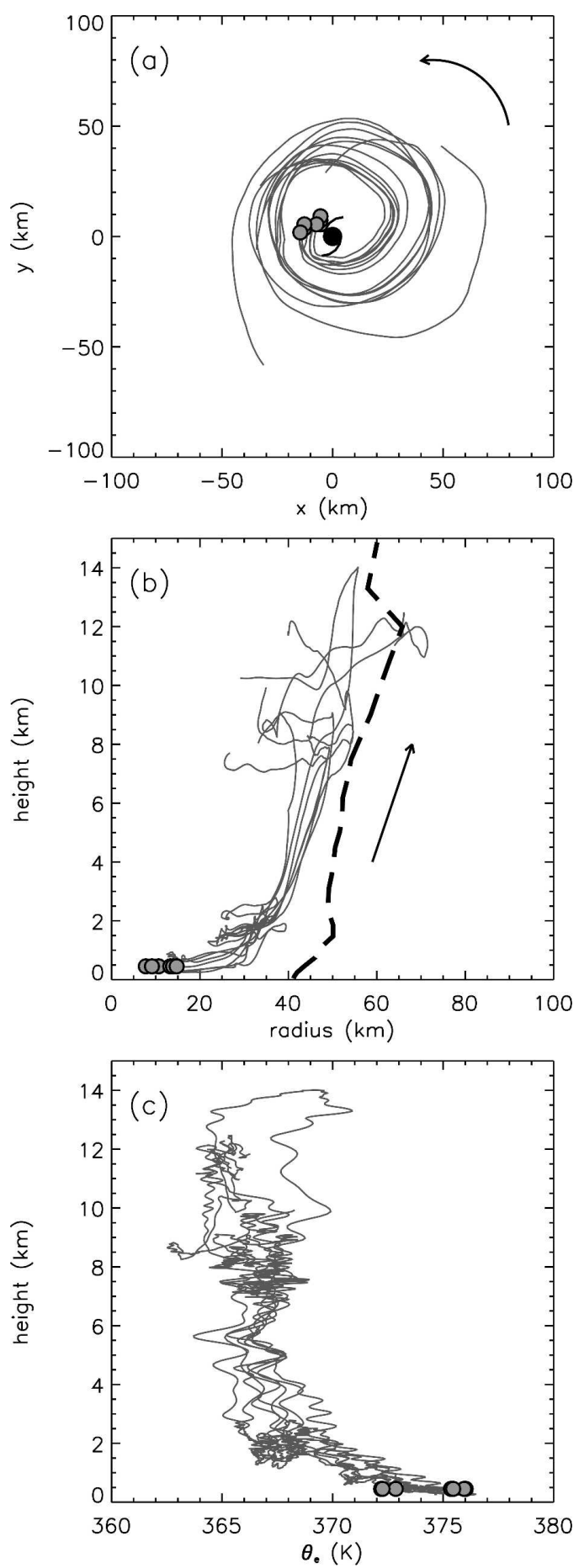

FIG. 5. Seven sample trajectories of class I (Fig. 1) viewed in (a) the horizontal $x-y$ plane and (b) the $r-z$ plane. Trajectories are seeded less than $15 \mathrm{~km}$ from the storm center and at a height of $453 \mathrm{~m}$. Trajectory seed time is $15 \mathrm{~h}$ into the $2-\mathrm{km}$ grid simulation and trajectories are calculated $5 \mathrm{~h}$ forward in time. (c) Associated values of $\theta_{e}$ as a function of height for the trajectories. Arrows indicate the direction of motion of the trajectories. The 5-h time average of the radius of maximum azimuthally averaged tangential wind as a function of height is plotted as a dashed line in (b). Seed locations are marked by light gray circles. along the trajectories becomes relatively constant with height above $z=2 \mathrm{~km}$. These parcels of air, originating in the low-level eye, retain a degree of warmth relative to other eyewall parcels, as is seen by plotting asymmetric $\theta_{e}$ along the trajectories (Fig. 6a). Asymmetries in this paper are defined by subtracting the axisymmetric mean $\theta_{e}$ field from the complete $\theta_{e}$ field. Below $z=$ $3 \mathrm{~km}$, asymmetric $w$ (Fig. 6b) is both negative and positive, the former being associated with the downward and inward motion exhibited in Fig. 5b. Asymmetric $w$ is positive between $z=3 \mathrm{~km}$ and $7 \mathrm{~km}$, with values as high as $10 \mathrm{~m} \mathrm{~s}^{-1}$. The asymmetric pictures of $\theta_{e}$ and $w$ suggest that air stirred into the eyewall from the lowlevel eye retains some degree of positive buoyancy relative to other default parcels of eyewall air that did not previously reside in the low-level eye. This relative buoyancy can be associated with the hot towers identified by Braun (2002) and Smith et al. (2005).

Consider now the complete subset of trajectories that make their way out to the axisymmetric eyewall (Fig. 7). There is an overall tendency for these trajectories to be stirred out into the eyewall downshear and left of the shear vector, and stronger tendency is shown for those trajectories (dark dots) that are stirred out near the base of the eyewall. Of the 551 trajectories that are seeded inside the eye at this level, 313 trajectories, or $56.8 \%$, are mixed out to the eyewall within $5 \mathrm{~h}$. Although the escape points shown are located all around the RMW azimuth ring, the majority of trajectories (Fig. 7b) encounter the eyewall at locations southeast, east, and northeast of the storm center. These findings are consistent with the results in Braun et al. (2006), which show that the main eyewall updrafts occur downshear and left of the mean layer vertical shear vector.

\section{3) EVIDENCE OF SUPERINTENSITY MECHANISM}

PM03 defined superintensity as a state where hurricane intensity exceeds the predicted maximum intensity of Emanuel's (1995) maximum potential intensity (E-PI) theory. The mechanism identified by PM03 in their axisymmetric modeling was transport of air from the low-level eye to the eyewall. The low-level eye air was enhanced in $\theta_{e}$ relative to the eyewall air; thus, its introduction to the base of the eyewall represented an additional source of heat for the hurricane heat engine cycle. In E-PI theory, the only source of heat for driving the hurricane circulation is the ocean beneath the eyewall through a wind-induced vertical heat exchange.

The representative sample of trajectories shown in Fig. 5 provides evidence of the superintensity mechanism operating in the MM5 Bonnie simulation. Once these sample trajectories are stirred out to the eyewall, 

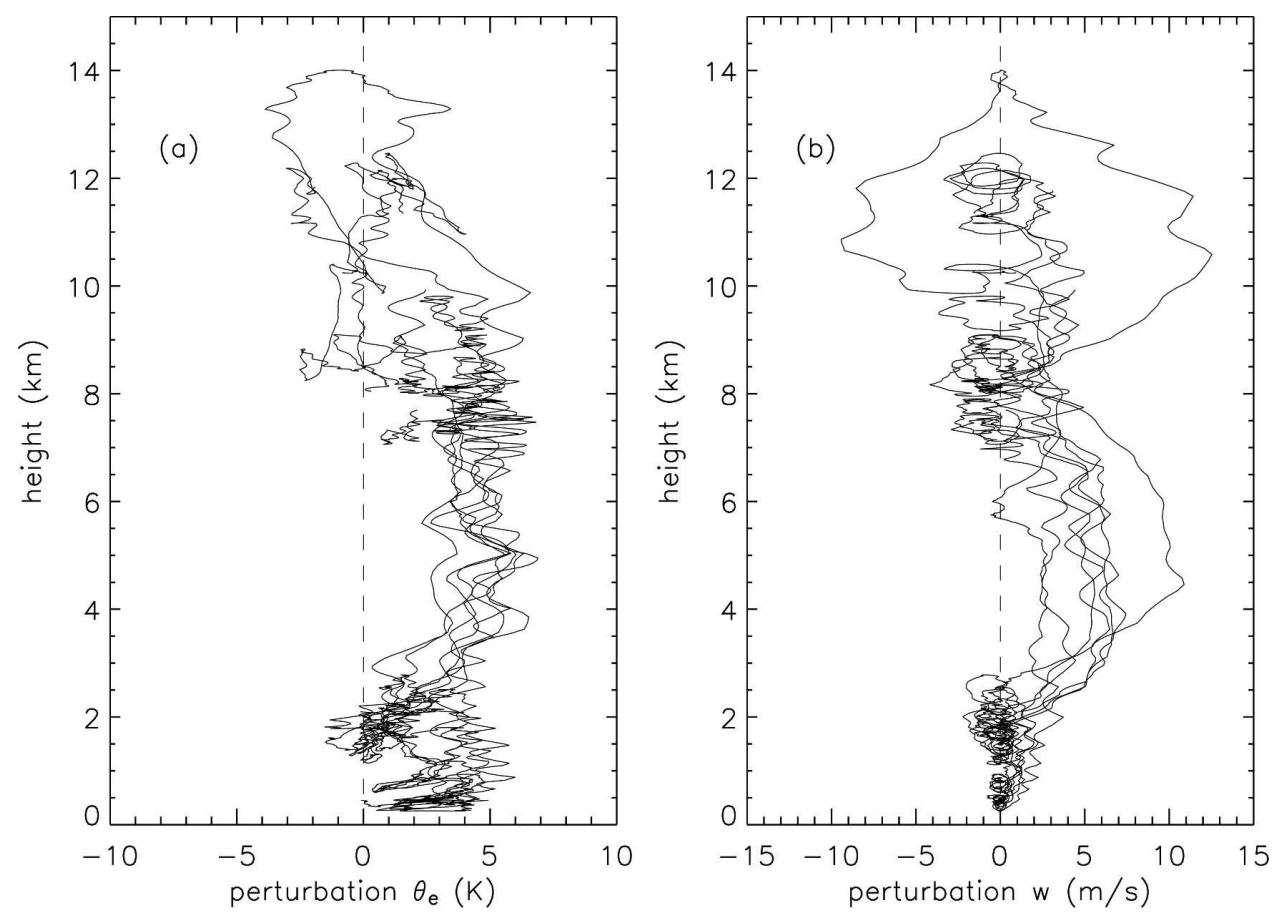

FIG. 6. (a) Asymmetric $\theta_{e}$ and (b) asymmetric $w$ as a function of height along the trajectories of class I (Fig. 1) shown in Fig. 5.

they are lifted by the eyewall updrafts. The form of the interaction between the relatively high- $\theta_{e}$ trajectory particles and the eyewall air is an incomplete reduction of trajectory $\theta_{e}$ to the mean eyewall value, retaining a moderate localized enhancement of the eyewall updraft associated with the trajectory (Fig. 6). Since $\theta_{e}$ is approximately conserved, ${ }^{4}$ a reduction of $\theta_{e}$ following the trajectory upon introduction to the eyewall must be associated with an increase in $\theta_{e}$ in the surrounding air, thus warming the axisymmetric mean $\theta_{e}$ by mixing.

The stirring of high $\theta_{e}$ air from the low-level eye into the eyewall is corroborated by instantaneous $r-z$ cross sections of $\theta_{e}$ along radial legs in the southeast quadrant of the storm (not shown). As shown in Braun et al. (2006), the eyewall updrafts originate in this quadrant of the storm, which corresponds to the downtilt-righthand side of the eyewall in relation to the environmental vertical shear vector. These cross sections of $\theta_{e}$ exhibit a tongue of high $-\theta_{e}$ air in the low-level eye extending into the base of the eyewall updrafts [see an

\footnotetext{
${ }^{4}$ The MM5 model is not designed to conserve $\theta_{e}$ of Bolton (1980). Both $\theta_{e}$ and MM5, however, are designed in reference to reality, so approximate conservation can be anticipated (and is roughly observed in the post analyses) in the absence of ice processes, surface interaction, or strong subgrid scale diffusive mixing.
}

example of this structure in Fig. 12 of Braun (2002)]. This suggests that this warmer $\theta_{e}$ air is being introduced to the eyewall updrafts as they form; it was not determined whether the introduction of the high $\theta_{e}$ air into the eyewall was the cause of the updraft initiation.

For the case of the Bonnie simulation, the sea surface temperature is $303 \mathrm{~K}$, the latitude is $24.2^{\circ} \mathrm{N}$, and the ambient surface pressure is $1010 \mathrm{mb}$. Since there is no dissipative heating in the numerical simulation, Emanuel (1995) represents the appropriate derivation of E-PI for comparison. A reasonable outflow temperature following the technique of PM03 of the axisymmetric circulation is $210 \mathrm{~K}$. To supply the values of surface relative humidity, we recall the closure assumption invoked by Emanuel (1995) that the relative humidity is hypothesized to have the same value at the eyewall as in the environment. We choose, therefore, $\mathcal{H}=80 \%$ as an expedient representative of the environment, in the spirit of the a priori approach of E-PI. Figure 4 shows, however, that relative humidity can be greater than $90 \%$ in the subcloud inflow layer. We thus also provide here a calculation using $\mathcal{H}=90 \%$ as representative of the eyewall, but we can anticipate a weaker magnitude of E-PI for a higher $\mathcal{H}$ (Camp and Montgomery 2001). Diagnosis of $C_{k} / C_{D}$ for the surface flux parameterization for this model run exhibits a spatial variability of the ratio, ranging from 0.65 for the 

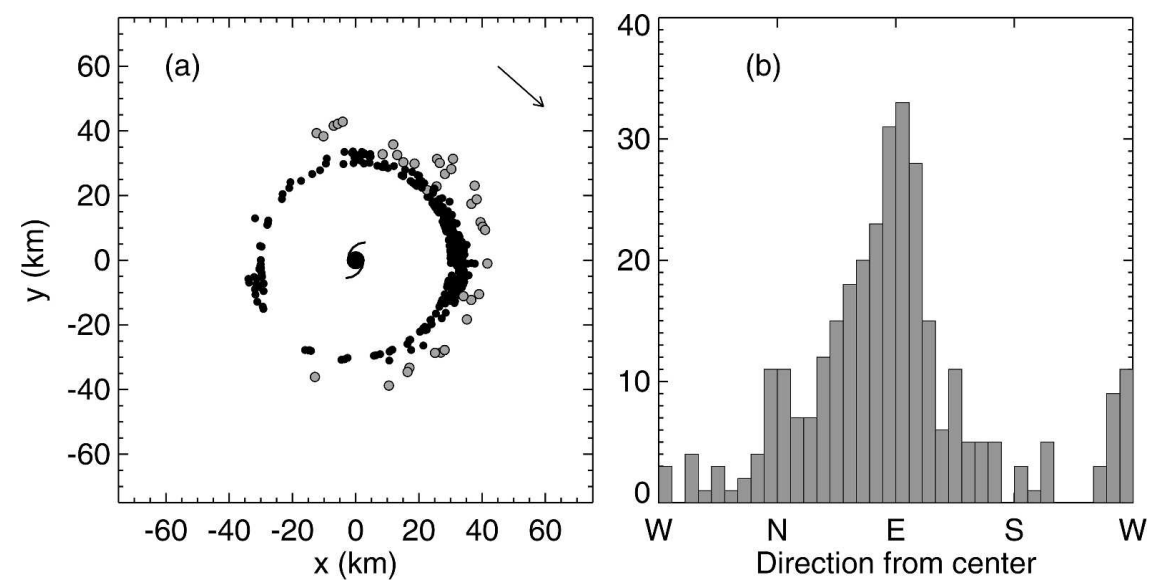

FIG. 7. (a) Locations where trajectories seeded inside the eye $\left(\bar{\theta}_{e}>370 \mathrm{~K}\right)$ at a height of $z=453 \mathrm{~m}$ (class I of Fig. 1) are stirred out to the axisymmetric eyewall. The arrow in the upper right corner indicates the direction of the mean layer $(850-200 \mathrm{mb}$ ) vertical shear vector. Eyewall encounters occurring at or below $z=1.5 \mathrm{~km}$ are denoted by dark circles, encounters above $z=1.5 \mathrm{~km}$ are light gray. (b) Histogram of the azimuths of the points shown in (a). Of the 551 total trajectories seeded inside the eye at this level, 313 trajectories $(57 \%)$ are stirred out and meet the eyewall criteria. Eye seed points extend out to a radius of $24 \mathrm{~km}$.

average over the complete inner domain to 0.35 at the radius of maximum winds. With these values and using $\mathcal{H}=80 \%$, E-PI predicts values of maximum $\bar{v}$ at the top of the boundary layer ranging from $52.2 \mathrm{~m} \mathrm{~s}^{-1}$ for $C_{k} / C_{D}=0.65$ to $38.2 \mathrm{~m} \mathrm{~s}^{-1}$ for $C_{k} / C_{D}=0.35$. For $\mathcal{H}=90 \%$, the values are $36.5 \mathrm{~m} \mathrm{~s}^{-1}$ for $C_{k} / C_{D}=0.65$ and $26.9 \mathrm{~m} \mathrm{~s}^{-1}$ for $C_{k} / C_{D}=0.35$. The strongest axisymmetric tangential winds of $55 \mathrm{~m} \mathrm{~s}^{-1}$ found at $(r=48$, $z=0.8 \mathrm{~km})$ in the Bonnie simulation are near to, or much greater than, E-PI. The simulation presented here is thus plausibly superintense. Considering that the simulated Bonnie presented here was under the influence of moderate vertical wind shear [which is associated with weaker intensity in the literature (DeMaria 1996; Frank and Ritchie 2001; Wu and Braun 2004)], it is noteworthy that the storm is near its E-PI value (or much stronger, depending on selection of parameters $)^{5}$

\footnotetext{
${ }^{5}$ In a strict sense, E-PI theory is valid for tropical cyclones in a steady-state configuration. Although the maximum tangential wind speed exhibits a near-steady tendency (Fig. 2), the minimum central pressure decreases nearly linearly with time, thus suggesting the simulated storm is not in a steady-state configuration during our analysis period. Given the thermodynamic conditions, one can nevertheless compare the modeled storm intensity to the comparable equilibrium intensity anticipated by theory. While we accept that transients could increase intensity stronger than E-PI for some short period of time, we suggest that only the eye-eyewall exchanges of warmth and moisture can sustain the storm at or stronger than E-PI for such a multihour period in the face of moderate vertical wind shear.
}

\section{b. Eye inflow trajectories and replenishment of the $\theta_{e}$ reservoir}

\section{1) The TRAJeCtORY SAMPLE}

If the eyewall updrafts are consuming some of the high $-\theta_{e}$ air within the low-level eye, then there must be some mechanism for replenishing that high $\theta_{e}$ reservoir. To examine this replenishment process, we investigate forward trajectories (class II of Fig. 1) seeded in the inflow layer converging on the storm center. The question to be addressed at this point is what percentage of the trajectories is transported underneath the eyewall updrafts and into the hurricane eye, as opposed to following the standard ascent path through the eyewall updrafts?

Seed points are located horizontally on a radius versus azimuth grid. Radial seed spacing is $5 \mathrm{~km}$, between $50<r<180 \mathrm{~km}$. Twenty-five azimuthal seed points exist at each seed radius. The vertical seed levels used are $z=40,121$, and $244 \mathrm{~m}$. This seeding strategy results in 625 trajectories calculated at each vertical seed level. The trajectories are calculated forward $3 \mathrm{~h}$ from a seed time of $t=15 \mathrm{~h}$ into the $2-\mathrm{km}$ simulation.

To ensure that trajectories seeded at or near the lowest model level $(z=40 \mathrm{~m})$ do not cross the lower grid boundary, it was necessary for us to extrapolate the velocity data to a near-surface height; we chose $z=10$ $\mathrm{m}$. Here we follow the logarithmic boundary layer as observed by Powell et al. (2003) ${ }^{6}$ and assign the mag-

\footnotetext{
${ }^{6} \mathrm{We}$ assume that the parameterization of the surface layer in the model follows similar assumptions.
} 

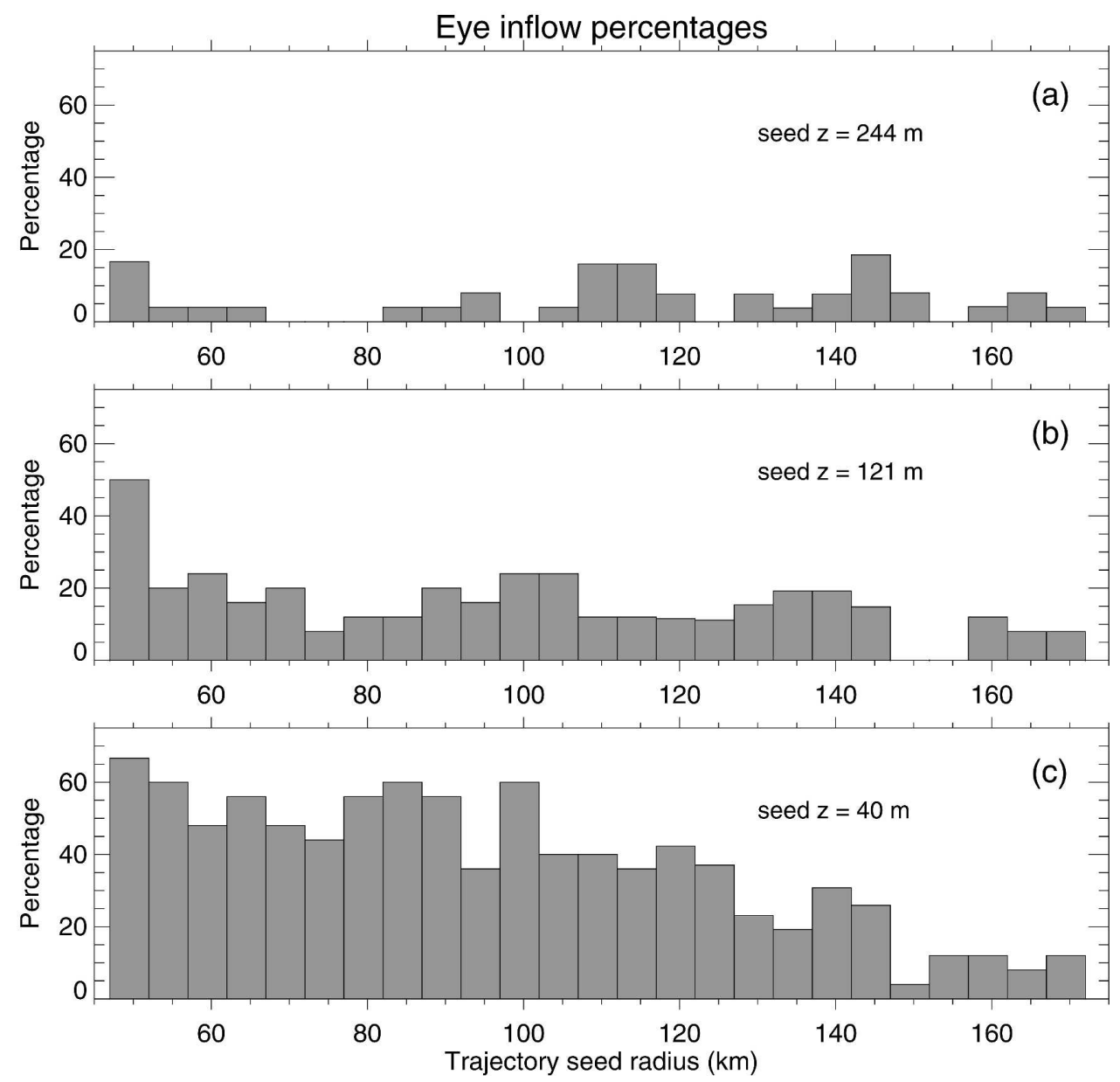

FIG. 8. Histogram of trajectories of class II (Fig. 1) that are transported into the eye, shown as a function of trajectory seed radius. Trajectory seed points for this analysis are every $5 \mathrm{~km}$ in the radial direction and seed levels of $z=40,121$, and $244 \mathrm{~m}$. Bin size is $5 \mathrm{~km}$ and sample size is 75 trajectories in each bin (25 at each seed level). Percentages are shown for (a) seed level $z=244 \mathrm{~m}$, (b) seed level $z=121 \mathrm{~m}$, and (c) seed level $z=40 \mathrm{~m}$. A trajectory is considered to be transported into the eye if its trajectory $\theta_{e}>370 \mathrm{~K}$ at $z<2 \mathrm{~km}$.

nitudes of the $u$ - and $v$-velocity components to be $85 \%$ of their values at $z=40 \mathrm{~m}$. We assign the vertical velocity $w$ to vanish at $z=10 \mathrm{~m}$ in order to ensure that no trajectories descend below $10 \mathrm{~m}$.

\section{2) LOW-LEVEL EYE MASS REPLACEMENT}

For a trajectory of class II to be transported into the eye, we required the $\theta_{e}$ value associated with a particular trajectory to exceed $370 \mathrm{~K}$ below a vertical height of $z=2 \mathrm{~km}$. This threshold value is chosen based on the values of $\theta_{e}$ in the high- $\theta_{e}$ reservoir of the low level eye (Fig. 4c). By this criterion, a significant fraction of class II trajectories (i.e., the boundary layer inflow) spends at least some time in the eye (Fig. 8). Considering trajectories originating just outside the eyewall $(r<100 \mathrm{~km})$, close to half of low level $(z=40 \mathrm{~m}$ seeding; Fig. $8 \mathrm{c})$ air is found to slip under the eyewall and enter the eye, with probability decreasing with height to about $20 \%$ at $z=121 \mathrm{~m}$. At further radii $(r>125 \mathrm{~km})$, turbulent redistribution between levels in the boundary layer makes the distinction between the percentages at these levels less definitive. Additionally, at larger radii, there is an increased chance that trajectories will be intercepted by the outer rain bands or that the 3 -h integration is insufficient to permit the inflow to reach the eyewall.

We now seek trajectories that can serve as prototypes for the two primary consequences for class II trajectories that reach the eyewall: either to enter the eye for some period of time or to be immediately drawn into the eyewall updraft without encountering the eye. The approach for constructing these prototypes will eliminate a large number of borderline cases, since these trajectories typify neither consequence. For the eye in- 

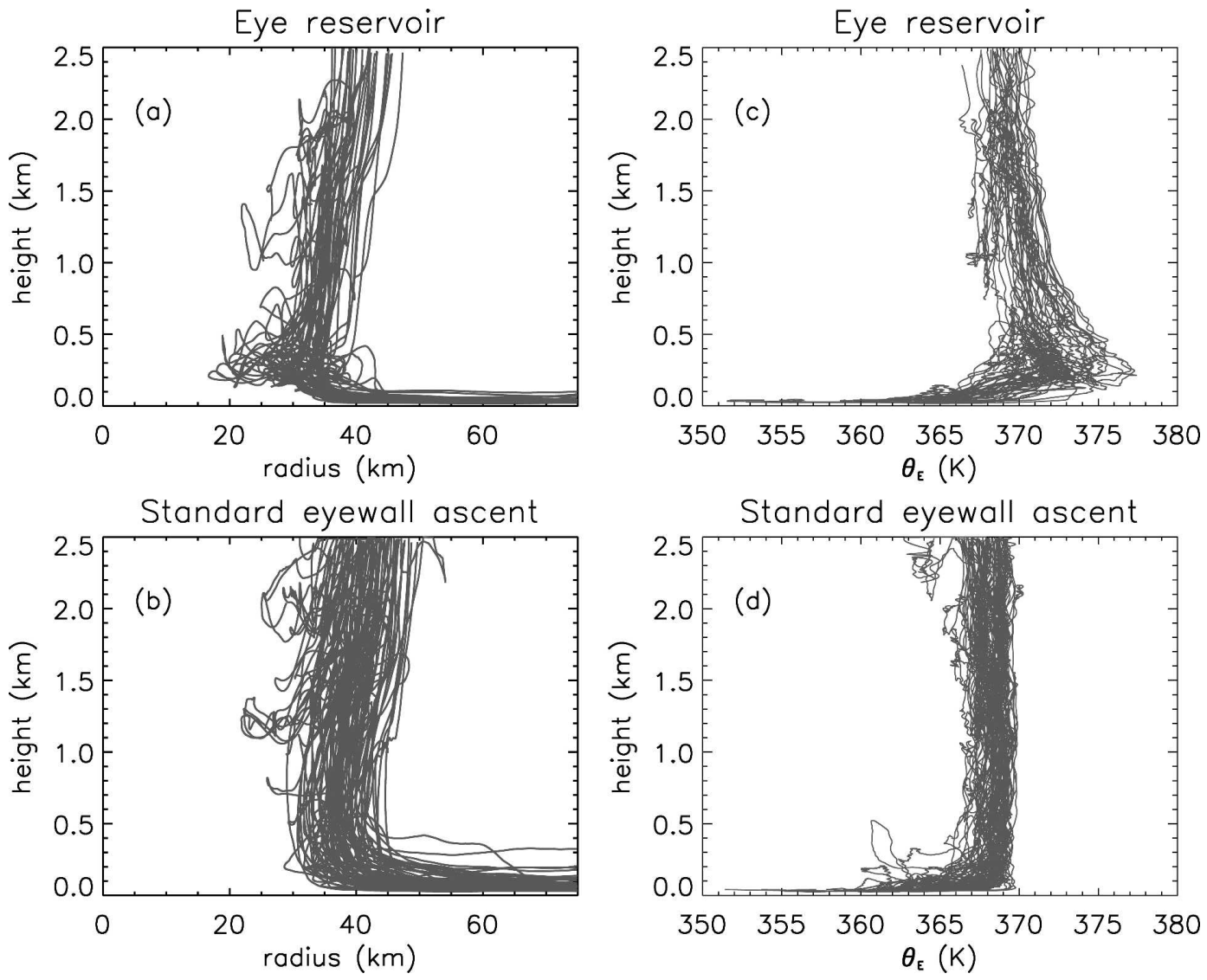

FIG. 9. Radius vs height plots for a select sample of class II (Fig. 1) trajectories that can be identified as (a) eye reservoir trajectories or (b) standard eyewall ascent trajectories, and (c),(d) $\theta_{e}$ vs height for the same trajectories. Sample sizes are 41 for eye reservoir and 78 for standard eyewall ascent.

flow prototypes, consider those trajectories of class II defined above which exceed $\theta_{e}=372 \mathrm{~K}$ at some point in the calculation. For the eyewall ascent prototypes, consider those trajectories of class II with maximum $\theta_{e}$ between $369 \mathrm{~K}<\theta_{e}<370 \mathrm{~K}$ (Fig. 9). The amount of time eye inflow prototype trajectories spend in the eye can vary greatly, where this time is defined as the period spent with $\theta_{e}>370 \mathrm{~K}$ just prior to entrainment into the eyewall updraft. The amount of time required for a trajectory to acquire a substantial increase in $\theta_{e}$ (3 K) can be as short as 15 min (Fig. 10), although 40-60 min is typical. The behavior described here is sufficient to explain how the eye entropy reservoir is maintained; the mass to replace air entrained into the eyewall can be adequately accounted for while it is demonstrated that the $\theta_{e}$ of these parcels can be quickly enhanced through exchange with the underlying ocean.

\section{3) SuPERinTENSity MeChanism MAintained}

The eye inflow trajectories (Figs. 9a,c) show clear evidence of the superintensity mechanism occurring.
All trajectories converge toward the center near the surface $(z<200 \mathrm{~m}$ at $r>40 \mathrm{~km}$ and $z \leq 500 \mathrm{~m}$ at $r<40 \mathrm{~km}$ ) and approach to within $15-25 \mathrm{~km}$ of the storm center as they are stirred into the eye. When they are stirred out to the eyewall and lifted by the

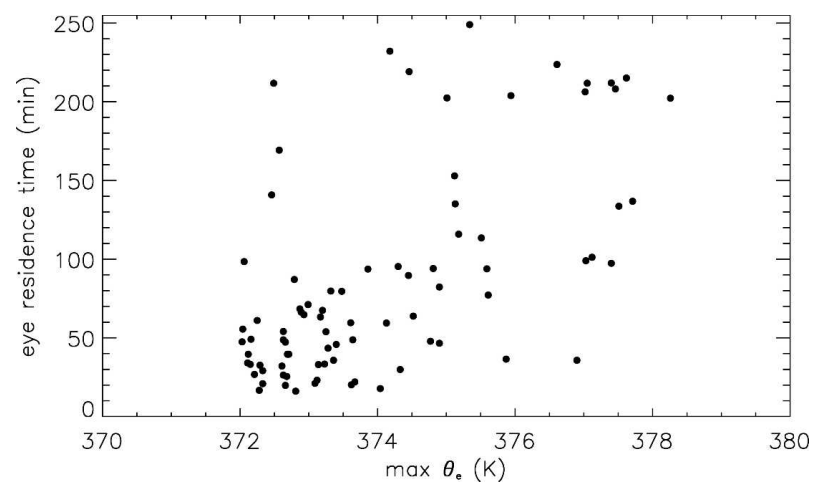

FIG. 10. Maximum $\theta_{e}$ vs eye residence time for the eye reservoir trajectories shown in Figs. 9a,c. These trajectories are of class II from Fig. 1. 


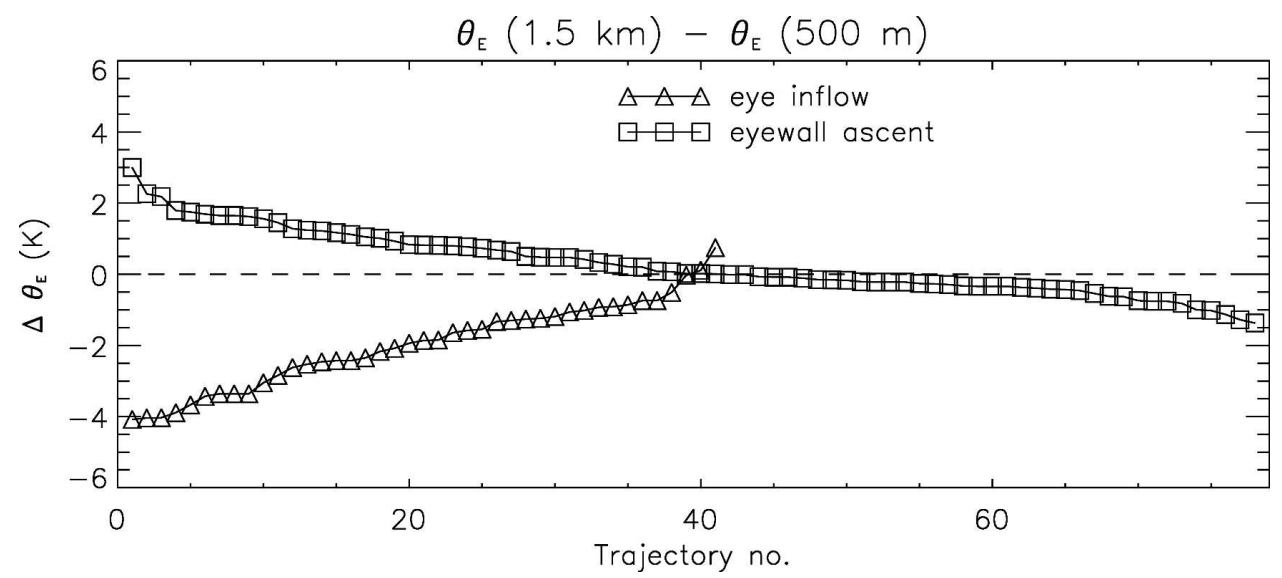

FIG. 11. Difference in $\theta_{e}$ between $z=1.5 \mathrm{~km}$ and $500 \mathrm{~m}\left[\Delta \theta_{e}=\theta_{e}(z=1.5 \mathrm{~km})-\theta_{e}(z=0.5 \mathrm{~km})\right]$ for the eye reservoir (triangles) and standard eyewall ascent (squares) trajectories shown in Figs. 9a,c. The numbering of trajectories along the $x$ axis is arbitrary. These trajectories are of class II from Fig. 1 .

eyewall updrafts (Fig. 9c), the interaction between the high- $\theta_{e}$ trajectory particles and the eyewall air is apparent. The $\theta_{e}$ value of the trajectories is not maintained at the values found in the low-level eye, but decrease partially to the value of the surrounding eyewall air as a result of diffusive mixing. Conversely, the eyewall ascent trajectories (Figs. 9b,d) show a slight warming initially in $\theta_{e}$ as they ascend in the eyewall updraft.

After exiting the eye, most of the eye inflow trajectories exhibit $\theta_{e}$ decreases of as much as $4 \mathrm{~K}$ (Fig. 11), as a result of mixing with relatively cooler eyewall air. The eyewall ascent trajectories, in turn, exhibit increases in $\theta_{e}$-up to $3 \mathrm{~K}$-resulting from their interaction with the higher $-\theta_{e}$ air coming from the low-level eye.

As the moist entropy of the low-level eye is stirred into to the eyewall, the reservoir of high- $\theta_{e}$ air in the low-level eye must be replenished. This is achieved primarily via the convergence of air toward the storm center in the low-level inflow layer as shown above (Fig. 9a).

\section{c. The upper-level eye-eyewall interaction}

\section{1) The trajectory sample}

To study the degree of isolation of the eye from the eyewall in the mid- to upper troposphere, we examine in this section forward trajectories (class III of Fig. 1) seeded within the eye above the inversion. The procedure follows section $3 \mathrm{a}$ except that seeding at heights of 1.1, 5.6, and $9.9 \mathrm{~km}$ will also be considered. The latter two are taken to represent air parcels from the mid- to upper-level eye, while $1.1 \mathrm{~km}$ is more representative of the lower-level eye.

\section{2) The COntainment Vessel hypothesis REVIEWED}

For trajectories originating inside the eye in the middle and upper troposphere, we are interested in whether the eye behaves as a containment vessel. Proposed by W98, the containment vessel hypothesis posits that the air contained inside the eye above the eye inversion in intense tropical cyclones has remained inside the eye since the eye formed. Using flight-level and dropsonde data, W98 identified eye soundings typical of intensifying tropical cyclones. The main features of these soundings included an eye inversion between 900 and $850 \mathrm{mb}$ and dewpoint depressions of approximately $10 \mathrm{~K}$ above the inversion. The rise and/or fall of the inversion results from a balance between low-level moist inflow, which is brought into the eye to compensate for the moist low-level eye air lost to the eyewall updrafts (as shown previously in section 3b), and warm, dry subsidence above the inversion induced by the loss of low-level mass to the eyewall updrafts. W98 describes the convective eyewall updrafts as heat pumps, which do work on the eye by drawing moist air out of the low-level eye and hence force thermally indirect descent above the eye inversion.

The eye soundings typical of weakening hurricanes (W98) showed eyewall moisture being mixed into the eye at mid- to upper levels. In contrast to intensifying storms, the weakening case is characterized by a more gradual Y-shaped inversion. The base of the inversion in the weakening cases is typically found (W98) between 850 and $700 \mathrm{mb}$. 


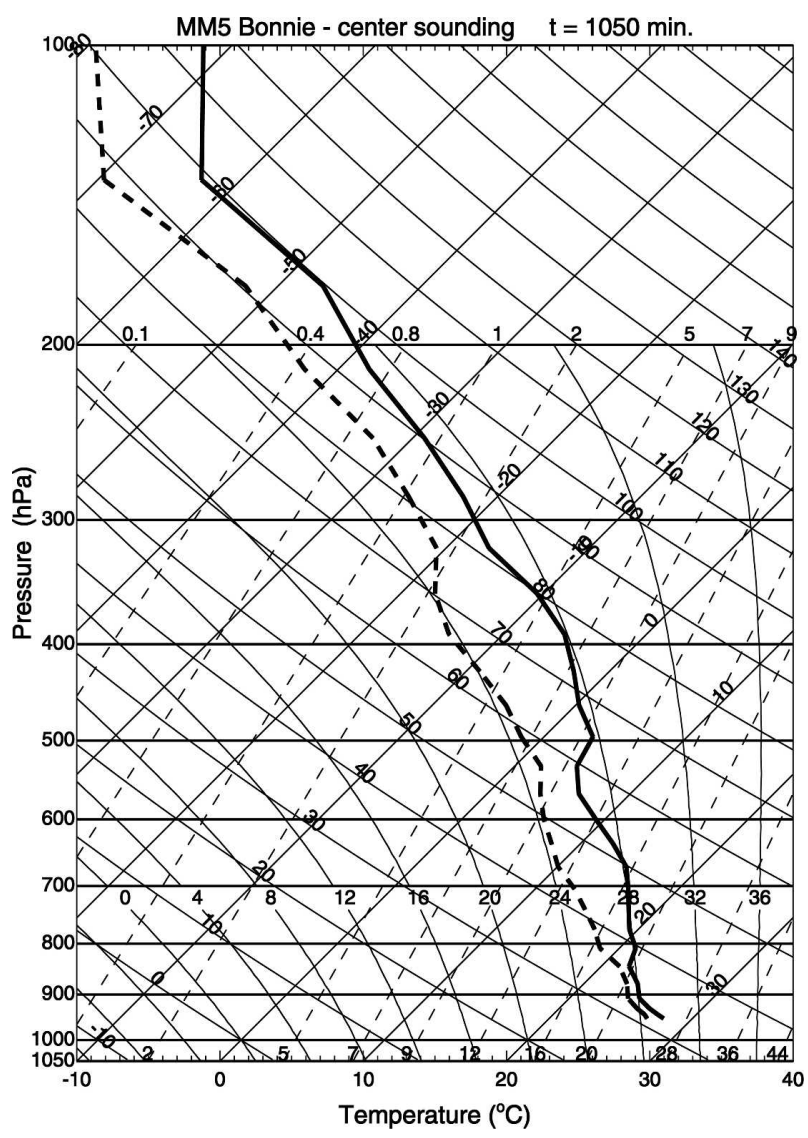

FIG. 12. Skew $T-\log p$ diagram of the sounding located inside the eye at the storm center. The time displayed is $t=17.5 \mathrm{~h}$, or halfway through the 5-h forward trajectory calculation. Temperature is the solid line and dewpoint is the dashed line.

\section{3) EVIDENCE OF UPPER-LEVEL EYE MASS RECYCLING}

The MM5 Bonnie simulation described in Braun et al. (2006) and herein appears to be more typical of the soundings in W98 in which eyewall moisture is being stirred into the eye (Fig. 12). The center eye sounding follows a saturated adiabat between 900 and $650 \mathrm{mb}$, with the exception of a shallow quasi-isothermal layer between 850 and $800 \mathrm{mb}$. The air between $850 \mathrm{mb}$ and the ocean is moister, with a fairly constant dewpoint depression of approximately $0.8 \mathrm{~K}$. Above the isothermal layer, dewpoint depressions range between 2 and $8 \mathrm{~K}$.

Trajectories seeded in the eye at the $z=5.6$ - and 9.8-km levels suggest that more than half $(59 \%$ at 5.6 $\mathrm{km}, 76 \%$ at $9.8 \mathrm{~km}$ ) of the eye air is removed from the axisymmetric eye to the axisymmetric eyewall (using the same criteria as above) over the 5-h period of study. At $z=5.6 \mathrm{~km}$, the exit locations for trajectories (Fig. 13) tend to be toward the southeast sector of the eye, consistent with the radial outflow shown at these azimuths (Fig. 3c). At $z=9.8 \mathrm{~km}$, the pattern is the same but is shifted toward southern azimuths (Figs. 14, 3d). Braun et al. (2006) has associated this radial wind signature with the outflow from strong updraft towers which form on the southern side of the eyewall.

Figure 15 shows the percentage of eye trajectories seeded at low- to midlevels $(z=453 \mathrm{~m}, 1.1 \mathrm{~km}$, and 5.6 $\mathrm{km})$ that are stirred into the eyewall. In general, the percentage increases with increasing seed radius, and
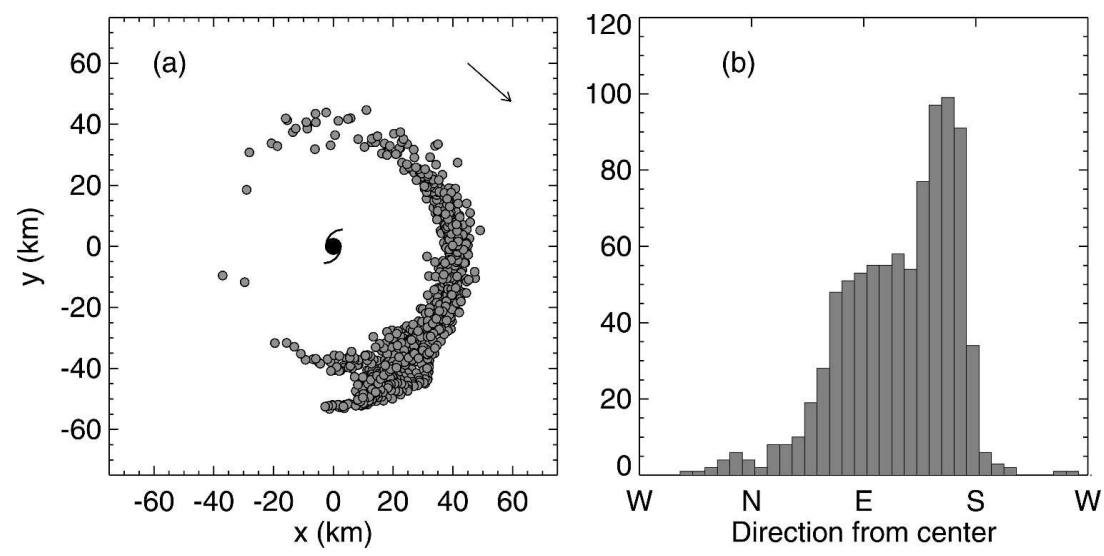

FIG. 13. (a) Locations where trajectories seeded inside the eye $\left(\bar{w}<0.25 \mathrm{~m} \mathrm{~s}^{-1}\right.$ and $\overline{\mathcal{H}}<$ $90 \%$ ) at a height of $z=5.6 \mathrm{~km}$ are stirred into the axisymmetric eyewall. The arrow in the upper right corner indicates the direction of the mean layer $(850-200 \mathrm{mb})$ vertical shear vector. (b) Histogram of the azimuths of the points shown in (a). Of the 1497 total trajectories seeded inside the eye at this level, 878 trajectories (59\%) are stirred out and meet the eyewall criteria. These trajectories are of class III from Fig. 1. Eye seed points extend out to a radius of $40 \mathrm{~km}$. 

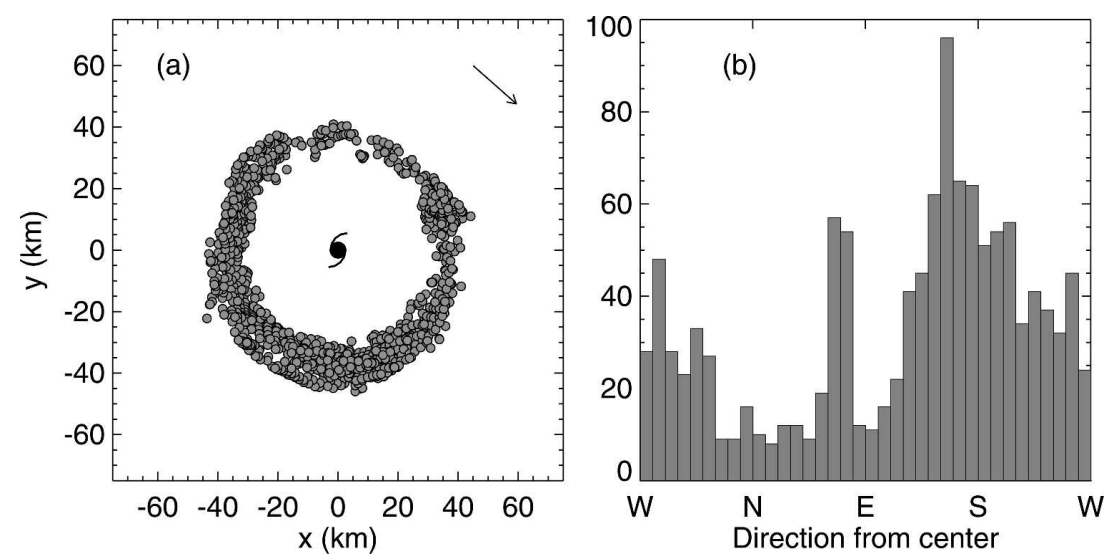

FIG. 14. As in Fig. 13 but at a height of $z=9.8 \mathrm{~km}$. Of the 1601 total trajectories seeded inside the eye at this level, 1210 trajectories $(76 \%)$ are stirred out and meet the eyewall criteria. Eye seed points extend out to a radius of $42 \mathrm{~km}$.

the percentage of trajectories seeded at lower levels $(z=453 \mathrm{~m}$ and $1.1 \mathrm{~km})$ that are being stirred into the eyewall is approximately $10 \%-20 \%$ higher than what is shown at the $z=5.6 \mathrm{~km}$ seed level. A large fraction ( $45 \%$ or more) of air from lower levels $(z=453 \mathrm{~m}$ and $1.1 \mathrm{~km}$ ) of the eye and within $r=15 \mathrm{~km}$ of the center encounters the eye within $5 \mathrm{~h}$, evidence for a large degree of mass replacement of the eye.

Although we have not comprehensively documented the mid- to upper-level eye replacement (which would require a longer time interval), the process is illustrated in part by the sample of seven class I trajectories (Fig. 5 ), where air rising through the eyewall detrains into the eye above $z=6 \mathrm{~km}$.

\section{d. Ventilation of the TC by the midlevel environment}

\section{1) The TRAJECTORY SAMPLE}

To examine the degree to which dry, midlevel environmental air intrudes upon the eyewall (ventilation) for this section, we investigate backward trajectories seeded across the eyewall and determine the sources of that air. All four classes of trajectories found in Fig. 1 can in principle be found in such a sample, and the objective will be to distinguish class IV trajectories from the sample and examine their thermodynamic characteristics.

The seed locations are at $z=5 \mathrm{~km}$ with equal radial $/$

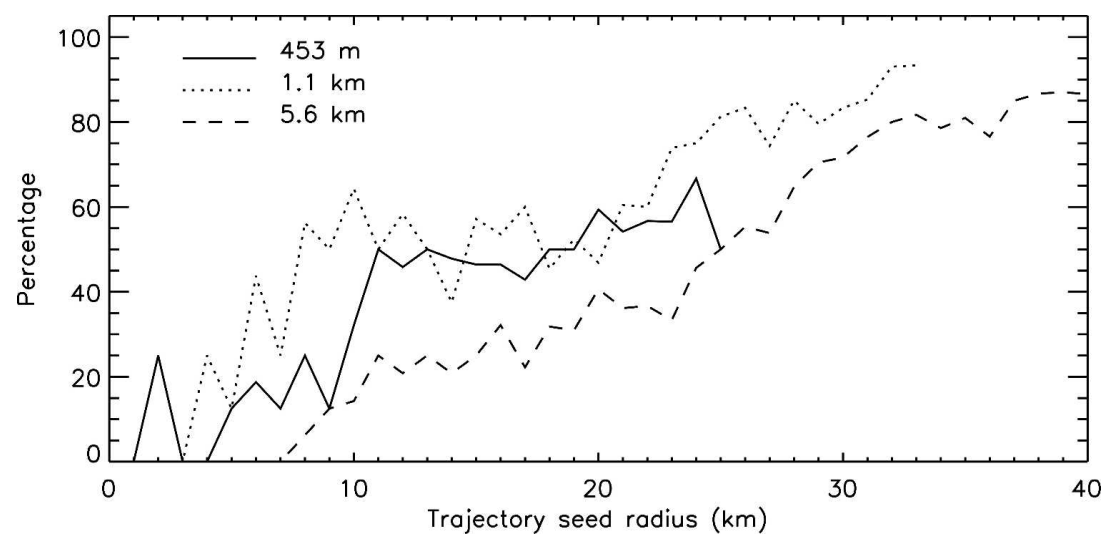

FIG. 15. Percentage of eye trajectories of class III seeded at each radius and at $z=453$ $\mathrm{m}$ (solid line), $1.1 \mathrm{~km}$ (dotted line), and $5.6 \mathrm{~km}$ (dashed line), which satisfy the criteria for being stirred into the axisymmetric mean eyewall $\left(\bar{w}>0.25 \mathrm{~m} \mathrm{~s}^{-1}\right.$ and $\left.\overline{\mathcal{H}}>90 \%\right)$. Percentages are plotted as a function of trajectory seed radius. Sample sizes are $551(453 \mathrm{~m})$, $903(1.1 \mathrm{~km})$, and $1497(5.6 \mathrm{~km})$ trajectories. Radial locations are binned to the nearest $1 \mathrm{~km}$. 

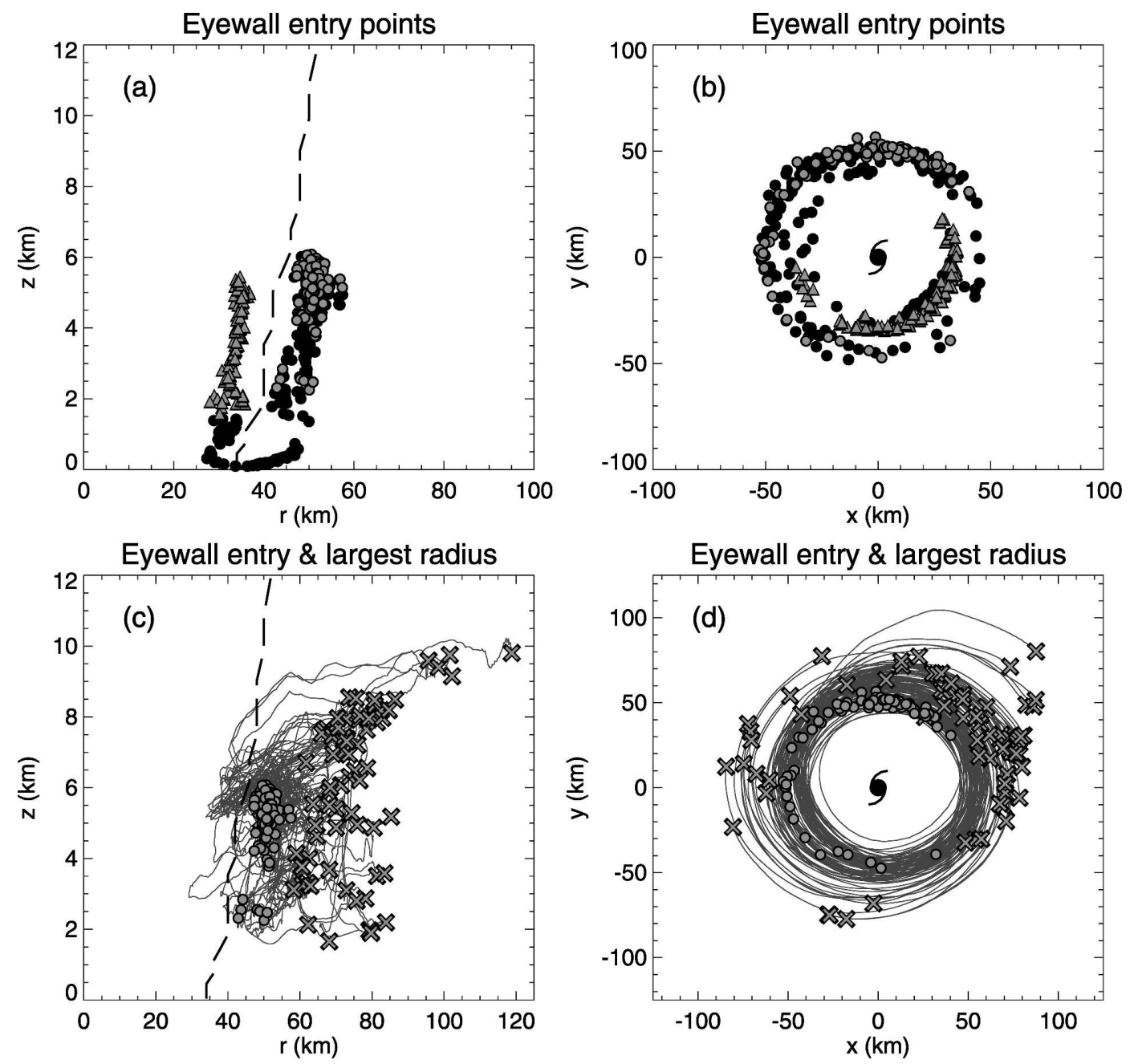

FIG. 16. (a) Locations where trajectories encounter the axisymmetric eyewall $\left(\bar{w}>0.25 \mathrm{~m} \mathrm{~s}^{-1}\right)$ plotted in the $(r, z)$ plane. Dark circles are trajectories determined to be class I or II (boundary layer inflow or low-level eye interaction; see Fig. 1); triangles are class III (mid- to upper-level eye interaction); and light circles are class IV (mid- to upper-level environmental interaction). (b) The same is shown in plan view. For each class IV trajectory, the eyewall entry point is again plotted with light circles and the point of farthest radius is shown with $\times$ 's (c) in an $(r, z)$ plane and $(\mathrm{d})$ as a plan-view plot, with trajectories overlaid. The dashed line is the radius of maximum updraft.

azimuthal spacing between $36<r<50 \mathrm{~km}$. There are 50 seed points at each radius, and the radial spacing is $2 \mathrm{~km}$, for a total of 400 trajectory seeds. The seed time used is $t=20 \mathrm{~h}$ and the backward calculation is carried out for $5 \mathrm{~h}$. Throughout the seeding annulus $\bar{w}>0.25$ $\mathrm{m} \mathrm{s}^{-1}$.

The process for distinguishing different classes of trajectories is as follows. For each back trajectory, $\bar{w}>$ $0.25 \mathrm{~m} \mathrm{~s}^{-1}$ at the seed (final) time, by construction. Each trajectory may be followed backward in time from the seed location to determine the first location where $\bar{w}$ is no longer greater than $>0.25 \mathrm{~m} \mathrm{~s}^{-1}$, which we define as the eyewall entry location. It is possible that one or more trajectories might remain in the axisymmetric eyewall throughout the trajectory calculation, but it was found that all trajectories had origins outside the eyewall through the 5-h calculation period. Trajectories with eyewall entry points below $z=1.5 \mathrm{~km}$ are considered to be in either class I or II, and no effort is made in the rest of this section to distinguish class I from class II. Trajectories with eyewall entry above $z=1.5 \mathrm{~km}$ but at a radius interior to the radius of maximum updraft are considered class III. For the rest of the trajectories (eyewall entry above $z=1.5 \mathrm{~km}$ and outside the radius of maximum updraft), two modes were identified (Fig. 16a). The first mode of trajectories were involved in updrafts just exterior to the axisymmetric eyewall (because of a noncircular shape of the eyewall) that 
emerged from the boundary layer; these were classified with the class I/II trajectories by their starting points being below $z=1.5 \mathrm{~km}$. The remainder of trajectories were then classified as class IV; thus these are trajectories with eyewall entry points above $z=1.5 \mathrm{~km}$, radii exterior to the radius of maximum updraft, and starting locations above $z=1.5 \mathrm{~km}$.

\section{2) ENVIRONMENTAL ENTRAINMENT}

Of the 400 trajectories, 213 are of class I/II, 115 are of class III, and 72 are of class IV. Class I/II trajectories are somewhat evenly distributed in azimuth (dark dots; Fig. 16b). The distribution of class III (triangles) and class IV (light dots; Fig. 16b) on the other hand show distinct azimuthal biases, apparently related to the 12 $\mathrm{m} \mathrm{s}^{-1}$ northwesterly shear across the system; class III entry points appear on the downshear and class IV entry points on the upshear azimuths of the storm. The locations where the class IV trajectories have their maximum radius can be used $(X$ symbols in Figs. 16c,d) to study the means of interaction from the environment. These maximum radius points are generally position to the left of the shear vector. Many of these trajectories quickly make their way to the eyewall, within a quarter rotation about the center; while others, whose maximum radii occur up to five full hours prior to the seed time (the maximum possible by this calculation), orbit more than 2 times before encountering the eyewall. Several of those trajectories that make the quick approach to the eyewall appear to descend in approach along a slanted path, while the rest appear to maintain a constant height during approach to the eyewall.

\section{3) Ventilation illustrated}

Ventilation is thought to weaken the hurricane, largely because of the transport of properties from the environment that are detrimental to maintenance of the hurricane. Our approach here is to investigate thermodynamic alterations of the eyewall by environmental air. The midtroposphere in the tropical environment typically shows a midlevel $\theta_{e}$ minimum (Holton 1992); in terms of $\theta_{e}$, the hurricane eye/eyewall are the warmest areas outside of the stratosphere. Any lateral mixing from the midtropospheric environment into the eyewall will reduce eyewall $\theta_{e}$ on average. Such a reduction in $\theta_{e}$ has been hypothesized to reduce the effectiveness of the hurricane heat engine (Riehl and Malkus 1961; Emanuel et al. 2004) and/or weaken the warm-core temperature anomaly aloft that supports the low surface pressure signature of the hurricane (Gray 1968; Frank and Ritchie 2001; Knaff et al. 2004).
TABLE 1. Midtropospheric environmental trajectory (class IV) statistics. The change in $\theta_{e}$ along the trajectories is $\Delta \theta_{e}=\theta_{e}(t=$ farthest radius $)-\theta_{e}(t=$ seed point $)$. Ensemble averages are denoted by $\langle\cdot\rangle, \sigma$ is standard deviation, prime symbols denote departures from the axisymmetric mean, and $\Delta \lambda$ represents the change in azimuth along the trajectory paths.

\begin{tabular}{lccc}
\hline \hline & $\Delta \theta_{e}<0$ & $\Delta \theta_{e}>0$ & \multicolumn{1}{c}{ All } \\
\hline$N$ & 32 & 40 & 72 \\
$\left\langle\Delta \theta_{e}\right\rangle$ & $-4.87 \mathrm{~K}$ & $2.30 \mathrm{~K}$ & $-0.89 \mathrm{~K}$ \\
$\sigma\left(\Delta \theta_{e}\right)$ & $4.39 \mathrm{~K}$ & $1.38 \mathrm{~K}$ & $4.72 \mathrm{~K}$ \\
$\left\langle\theta_{e}^{\prime}\right\rangle$ (seed point $)$ & $-1.30 \mathrm{~K}$ & $-5.20 \mathrm{~K}$ & $-3.47 \mathrm{~K}$ \\
$\sigma\left(\theta_{e}^{\prime}\right)$ & $3.79 \mathrm{~K}$ & $2.31 \mathrm{~K}$ & $3.60 \mathrm{~K}$ \\
$\left\langle\Delta \theta_{e}\right\rangle+\left\langle\theta_{e}^{\prime}\right\rangle$ & $-6.17 \mathrm{~K}$ & $-2.90 \mathrm{~K}$ & $-4.36 \mathrm{~K}$ \\
$\langle\Delta \lambda\rangle$ & $8.44 \mathrm{rad}$ & $7.76 \mathrm{rad}$ & $8.06 \mathrm{rad}$ \\
$\sigma(\Delta \lambda)$ & $3.29 \mathrm{rad}$ & $2.58 \mathrm{rad}$ & $2.92 \mathrm{rad}$ \\
\hline
\end{tabular}

The simplest measure of ventilation of eyewall air with environmental air is to note that 72 of 400 trajectories, representing $18 \%$ of the mass (and number, coincidentally) rising through $z=5 \mathrm{~km}$ in the eyewall, originated in the environment (light colored dots; Fig. 16). The large number of interacting trajectories would appear to be a consequence of analyzing a sheared hurricane simulation. While a direct comparison of mixing processes in a three-dimensional simulation and axisymmetry must be done cautiously, we performed a comparative axisymmetric simulation using the $4 \mathrm{x}$ run from PM03 (which is necessarily without vertical wind shear; not shown) and found zero class IV trajectories. We plan, in future work, to extend the present analysis to idealized, three-dimensional hurricane simulations without vertical shear.

An analysis of entrant class IV trajectories is summarized in Table 1 . Here, the change in $\theta_{e}$ along the trajectory is measured using

$$
\Delta \theta_{e}=\theta_{e}(t=\text { farthest radius })-\theta_{e}(t=\text { seed point })
$$

and the dataset is partitioned according to whether $\Delta \theta_{e}$ is positive or negative. When $\Delta \theta_{e}<0$, the trajectory warms on approach to the eyewall, which must come at the cost of a reduction of $\theta_{e}$ from the eyewall and the surrounding region, either through warming or moistening of the newly arriving trajectory. On average, class IV trajectories show negative values $\left(\left\langle\Delta \theta_{e}\right\rangle=-0.89 \mathrm{~K}\right.$; where $\langle\cdot\rangle$ is used for an ensemble average), indicating a net warming of such parcels upon approach to and entrance into the eyewall by some diffusive or irreversible mixing. Despite this warming, these class IV trajectories remain cool relative to the axisymmetric mean $\left(\theta_{e}^{\prime}<\right.$ $0)$. Also shown in Table 1 are standard deviations $\sigma$ of $\Delta \theta_{e}$ and $\theta_{e}^{\prime}$. Eyewall $\theta_{e}$ is reduced by the introduction of 
class IV trajectories, either directly by stirring cold air into the vicinity of the warmer eyewall air that emerges from the boundary layer inflow and the eye, or indirectly by diffusive mixing. Since class IV represents about $18 \%$ of the mass at $z=5 \mathrm{~km}$, the axisymmetric mean eyewall is cooled

$$
\begin{aligned}
\Delta \theta_{e}(\text { eyewall }) & \approx \frac{0.18}{1.0-0.18}(-0.89-3.47) \mathrm{K} \\
& \approx-0.96 \mathrm{~K} .
\end{aligned}
$$

The large standard deviations and the unbalanced sample size of positive and negative $\Delta \theta_{e} \mathrm{~s}$ are indicative of a positively skewed distribution, where, while all class IV parcels stir in mass, a small number of events may perform the largest modification of the eyewall mean $\theta_{e}$.

The technique of this section was applied using a second seed time (not shown), $t=19 \mathrm{~h}$ instead of $20 \mathrm{~h}$ used above. At this time, the axisymmetric eyewall was narrower, so the seed annulus was $38<r<48 \mathrm{~km}$. This provides 300 trajectories for analysis, 81 of which (27\%) were identified as class IV, thus there were more ventilating air parcels at this time. On the whole, this set of trajectories differs from the set illustrated above by a net decrease of entropy on approach to the eyewall $\left(\left\langle\Delta \theta_{e}\right\rangle=0.56 \mathrm{~K}\right.$ instead of $\left.-0.89 \mathrm{~K}\right)$ with less variance $\left[\sigma\left(\Delta \theta_{e}\right)=3.86 \mathrm{~K}\right.$ instead of $\left.4.72 \mathrm{~K}\right]$, but a cooler asymmetric entropy at the seed point $\left(\left\langle\theta_{e}^{\prime}\right\rangle=-4.34 \mathrm{~K}\right.$ instead of $-3.47 \mathrm{~K})$, and this set of trajectories approaches the eyewall more abruptly (change in azimuth $\langle\Delta \lambda\rangle=4.15$ rad versus $8.06 \mathrm{rad}$ ). Following the approach of (2) above, the axisymmetric mean eyewall can be found at this time to cool

$$
\begin{aligned}
\Delta \theta_{e}(\text { eyewall }) & \approx \frac{0.27}{1.0-0.27}(+0.56-4.34) \mathrm{K} \\
& \approx-1.40 \mathrm{~K} .
\end{aligned}
$$

The degree of eyewall cooling was found to be somewhat more consistent with the greater number of class IV trajectories.

\section{Summary and conclusions}

This study builds on the numerical study of Hurricane Bonnie by Braun et al. (2006) by examining the interactions between the various air masses (eye, eyewall, inflow, and environment) of the TC. Through analysis of parcel trajectories, we have been able to demonstrate that the low-level eye air can be carried into the eyewall; that this low-level eye air is replenished by inflow under the eyewall; that the mid- to upper-level eye air can be exchanged with eyewall air in a two-way process; and that mid- to upper-level air exterior to the eyewall can ventilate the eyewall (in the sense of Simpson and Riehl 1958; Riehl and Malkus 1961; Frank and Ritchie 2001; Emanuel et al. 2004).

The trajectories examined demonstrate that the superintensity mechanism as described by PM03 and Braun (2002) is active in the Bonnie simulation. The results suggest that the superintensity mechanism is still able to benefit the intensity of the storm while it is being sheared by the environment. Trajectories originating in the low-level eye and possessing relatively high $\theta_{e}$ are stirred into the base of eyewall updrafts and introduce an additional source of heat to the eyewall. The low-level eye is replenished by boundary layer parcels that slip underneath the eyewall updrafts to linger for some time in the eye (typically 40-60 min), thereby acquiring an elevated $\theta_{e}$ through prolonged interaction with the ocean surface. The stirring at low levels between the eye and eyewall occurs predominately left of the mean deep-layer environmental shear vector. Our results are consistent with the analysis of updrafts in this MM5 simulation conducted by Braun et al. (2006), and are also supported by the observational studies of Molinari et al. (2004) and Corbosiero (2004), the model results of Frank and Ritchie (1999, 2001), and the theoretical analysis by Reasor et al. (2004). The results in Bender (1997) also exhibited asymmetries forced by imposed vertical shear, though the rainfall in his study occurred preferentially upshear rather than left of the shear vector.

More than half of trajectories originating inside the eye in the middle and upper troposphere are not being contained inside the eye in our 5-h trajectory calculation period. More eye-eyewall mixing occurs in the upper troposphere than does in the hurricane boundary layer and middle $(z=5.6 \mathrm{~km})$ troposphere. For mature hurricanes in low vertical wind shear conditions, W98 hypothesized that eye containment of air parcels above the eye inversion would be quite strong. Our case is more consistent with the weakening cases of W98 and Carsey and Willoughby (2005). Containment of air within the eye is compromised in our case by stirring of air from the eyewall into the eye, but very little air is stirred into the eye from the environment above the boundary layer. Although a comprehensive analysis of mass replacement to the eye at mid- to upper levels was not carried out, there is evidence of eyewall air being stirred inward into the eye at these levels (e.g., Fig. 5).

Ventilation (Riehl and Malkus 1961; Emanuel et al. 2004) is illustrated in the Bonnie simulation, as trajectories track the migration of environmental air with low $\theta_{e}$ into the eyewall. The net impact is about a $1-\mathrm{K}$ re- 
duction of eyewall average $\theta_{e}$ based on analysis of air at $z=5 \mathrm{~km}$ in the eyewall for two instances in time. For this simulation of a vertically sheared hurricane, the intensity is plausibly much greater than E-PI (simulated 55 vs E-PI 38.2 to $52.2 \mathrm{~m} \mathrm{~s}^{-1}$ ). The interaction of environmental air with low entropy and the eyewall is hypothesized to weaken the TC. This is supported by the modeling study of Frank and Ritchie (2001), in which eddy mixing between the eye and eyewall was hypothesized to result in ventilation of the eye in the upper troposphere and subsequent weakening of their modeled TC.

One caveat to consider when interpreting the results presented here is that the mesoscale model used in this study is not capable of resolving all the physical processes relevant to the eye-eyewall mixing problem in TCs. Subgrid-scale diffusion parameterizations used in most numerical models are not applied with any realistic expectation of representing subgrid-scale turbulence in the atmosphere, rather they are used as a means to maintain numerical stability in the model. This particular simulation using MM5, for example, employs the Blackadar boundary layer scheme, which parameterizes subgrid-scale turbulence (in addition to boundary layer mixing) on the vertical structure only (MM5 does not include any horizontal mixing terms in the boundary layer scheme; lateral mixing occurs only through numerical diffusion). One thus cannot expect the model to realistically represent subgrid-scale mixing that would occur in the real atmosphere, and this caveat should be kept in mind when interpreting the Lagrangian results presented in this study. Additionally, although the 2-km inner grid simulation is capable of providing a representation of the mesoscale eddies occurring in the TC inner core, this horizontal resolution is too coarse to represent the larger turbulent eddies of the Kolmogorov inertial range. Using a model with horizontal grid spacing of 500-2000 m would still not suffice, since the effective Reynolds number (as defined in Bryan et al. 2003) of the flow at this resolution would not be large enough to allow an explicit calculation of the turbulence. The model configuration used in this study is the current state of the art, however, and the authors look forward to studies similar to this one being carried out on models capable of resolving the TC inner-core mixing processes. Computing power has advanced to the point where a large eddy simulation, with a horizontal grid resolution of $200 \mathrm{~m}$ or finer, could realistically be implemented in a TC simulation such as the one presented in this study.

Future work will consider several of the questions raised herein in more detail. Specifically, how substantial is $1-\mathrm{K}$ cooling in the eyewall in reducing the inten- sity of the TC? A continued study of sheared storms in an idealized setting is ongoing to examine the efficiency of ventilation for various magnitudes of shear. Finally, it remains to identify the principal asymmetric structures around the storms that contribute the most to its ventilation.

Acknowledgments. This research is supported in part by NASA CAMEX-4 Grant NAG5-11140, ONR Grant N00014-02-1-0474, and NSF Grants ATM-0132006, ATM-0349980, and ATM-0530884. Discussions with Kerry Emanuel helped improve both thought and presentation.

\section{APPENDIX A}

\section{Determination of Storm Center}

The technique for finding the storm center described below basically attempts to place the maximum radial gradient in surface pressure close to the same radius from the center of the storm at all azimuths. The alternative method of using the location of minimum surface pressure was found to track centers of mesovortices rather than the storm-scale center of pressure. The technique described below is designed to avoid any arbitrary selection of scale (the implicit scale is the spatial scale over which the signature of the gradient of surface pressure is distributed) and any iterative processes for solution.

The method for finding the storm center is as follows. Consider each grid point of a select subdomain of an $x-y$ grid of simulated surface pressure. Consider each of four rays radiating along the four cardinal directions from each point. Obviously, grid points near the edge of the defined grid will lack data in one or two directions. For this reason, consider our select subdomain to be the middle $1 / 9$ th of the domain; that is,

$$
\begin{aligned}
& \frac{N_{x}}{3} \leq I_{x}<\frac{2 N_{x}}{3} \\
& \frac{N_{y}}{3} \leq I_{y}<\frac{2 N_{y}}{3},
\end{aligned}
$$

where $I_{x}$ and $I_{y}$ are the index number of a grid point in the $x$ and $y$ directions, and $N_{x}$ and $N_{y}$ are the total number of grid points in the $x$ and $y$ directions, respectively. Determine the minimum $M$ of the number of grid points lying along each ray. Along each ray, a profile of surface pressure $P_{j}$ can be found, where $j \in\{1,2$, $3,4\}$ is an index for each of the four directions. Determine a score for each grid point $S_{x y}$ by a least mean squares difference from their mutual average 


$$
s=\frac{\sum_{j} \sum_{k=0}^{M} P_{j}(k)-\bar{P}(k)}{M},
$$

where

$$
\bar{P}=\frac{\sum_{j} P_{j}}{4}
$$

is the averaged pressure profile. Select the point where $s$ is a minimum as the storm center.

This method works because we know the innermost grid of the numerical model tracks the movement of the central surface pressure minimum, thus we are assured that the center will be found within the middle $1 / 9$ of the domain. Also, the scale of the vortex is somewhat larger than the scale of the inner-mesh grid. When the vortex scale is much smaller (such as on the coarsest grid mesh of our model run) than the size of the grid, then the four profiles might easily miss the vortex entirely and the score will measure differences in the more-or-less flat environmental surface pressure field, providing a small score trivially.

We may take $M$ above to be a fixed constant sufficiently small to handle every possible profile; that is,

$$
M=\min \left(\frac{N_{x}}{3}, \frac{N_{y}}{3}\right),
$$

but we found that change did not alter the resulting centerfind for our case.

\section{APPENDIX B}

\section{Computation of Trajectories}

A trajectory is the computation of the path of a hypothetical fluid particle through a specified flow field

$$
\frac{d \mathbf{r}_{m}(t)}{d t}=\mathbf{V}\left(\mathbf{r}_{m}, t\right),
$$

where $\mathbf{r}_{m}$ is the position of the trajectory of index $m$ as a function of time and $\mathbf{V}$ is the Eulerian velocity field as a function of space and time. The trajectories are computed using a second-order Runge-Kutta method (Press et al. 1992) where four-dimensional (space-time) linear interpolation is used to compute values of the wind field that generally fall between spatial/temporal grid points. This simple problem is complicated by the shifting of the inner grid as it moves with the storm center. By modeling practice, the shifting of the grid moves not by one grid increment frequently, but by increments of several grid points occasionally. To solve this problem, a four-dimensional universal grid large enough to contain the complete translation of the grid as it follows the storm is created, and all information is remapped to this grid. Naturally, much of this universal grid is empty, and no effort is made to populate these empty areas with other information available to us. Any trajectory leaving the inner (moving) mesh of the MM5 simulation is simply identified and considered to have left the near-storm environment.

The grid stagger of the wind fields is eliminated by interpolating the $u, v$, and $w$ wind fields to the $\theta$ or thermodynamic grid points, which is where the universal grid is defined. The winds are translated from $\mathrm{m} \mathrm{s}^{-1}$ to the units of (grid points) $\mathrm{s}^{-1}$, taking into account the effects of latitude using the formulas

$$
\begin{aligned}
& u_{\text {grid }}=\frac{u}{\Delta x}=\frac{u}{\cos (\phi)|\Delta \lambda| r_{\oplus}} \\
& v_{\text {grid }}=\frac{v}{\Delta y}=\frac{v}{(\Delta \phi) r_{\oplus}},
\end{aligned}
$$

where $u_{\text {grid }}$ and $v_{\text {grid }}$ are wind speeds in grid units, $\phi$ and $\lambda$ and latitude and longitude, respectively, converted to radians and $r_{\oplus}$ is the mean radius of the earth. Calculation in terms of grid spacing per unit time is a coding convenience that allows a general-purpose, generic, well-tested trajectory routine to be used without being required to manage details of spherical geometry. If the geographic pole were included, we can expect the trajectory code to fail there unless a more appropriate model grid system were used.

Since the trajectories are computed in earth-relative coordinates, storm motion is not subtracted from the winds, but the resulting trajectories can be renavigated to storm-centered coordinates. This renavigation was carried out for all trajectories shown in this study.

\section{REFERENCES}

Bassom, A. P., and A. D. Gilbert, 1999: The spiral wind-up and dissipation of vorticity and a passive scalar in a strained planar vortex. J. Fluid Mech., 398, 245-270.

Bender, M. A., 1997: The effect of relative flow on the asymmetric structure in the interior of hurricanes. J. Atmos. Sci., 54, 703724.

Black, M. L., J. F. Gamache, F. D. Marks Jr., C. E. Samsury, and H. E. Willoughby, 2002: Eastern Pacific Hurricanes Jimena of 1991 and Olivia of 1994: The effect of vertical shear on structure and intensity. Mon. Wea. Rev., 130, 2291-2312.

Bolton, D., 1980: The computation of equivalent potential temperature. Mon. Wea. Rev., 108, 1046-1053.

Braun, S. A., 2002: A cloud-resolving simulation of Hurricane Bob (1991): Storm structure and eyewall buoyancy. Mon. Wea. Rev., 130, 1573-1592.

_, M. T. Montgomery, and Z. Pu, 2006: High-resolution simulation of Hurricane Bonnie (1998). Part I: The organization of eyewall vertical motion. J. Atmos. Sci., 63, 19-42. 
Bryan, G. H., J. C. Wyngaard, and J. M. Fritsch, 2003: Resolution requirements for the simulation of deep moist convection. Mon. Wea. Rev., 131, 2394-2416.

Camp, J. P., and M. T. Montgomery, 2001: Hurricane maximum intensity: Past and present. Mon. Wea. Rev., 129, 1704-1717.

Carnevale, G. F., J. C. McWilliams, Y. Pomeau, J. B. Weiss, and W. R. Young, 1991: Evolution of vortex statistics in 2-dimensional turbulence. Phys. Rev. Lett., 66, 2735-2737.

Carsey, T. P., and H. E. Willoughby, 2005: Ozone measurements from eyewall transects of two Atlantic tropical cyclones. Mon. Wea. Rev., 133, 166-174.

Charney, J. G., 1973: Planetary fluid dynamics. Dynamic Meteorology, P. Morel, Ed., Reidel, 97-351.

Corbosiero, K. L., 2004: The structure and evolution of a hurricane in vertical wind shear: Hurricane Elena (1985). Preprints, 26th Conf. on Hurricanes and Tropical Meteorology, Miami, FL, Amer. Meteor. Soc., CD-ROM, 4A.2.

DeMaria, M., 1996: The effect of vertical shear on tropical cyclone intensity change. J. Atmos. Sci., 53, 2076-2087.

_, and J. Kaplan, 1994: A Statistical Hurricane Intensity Prediction Scheme (SHIPS) for the Atlantic basin. Wea. Forecasting, 9, 209-220.

Dudhia, J., 1993: A nonhydrostatic version of the Penn StateNCAR Mesoscale Model: Validation tests and simulation of an Atlantic cyclone and cold front. Mon. Wea. Rev., 121, 1493-1513.

Emanuel, K., 1986: An air-sea interaction theory for tropical cyclones. Part I: Steady-state maintenance. J. Atmos. Sci., 43, 585-605.

_ 1989: The finite-amplitude nature of tropical cyclogenesis. $J$. Atmos. Sci., 46, 3431-3456.

1995: Sensitivity of tropical cyclones to surface exchange coefficients and a revised steady-state model incorporating eye dynamics. J. Atmos. Sci., 52, 3969-3976.

- 1997: Some aspects of hurricane inner-core dynamics and energetics. J. Atmos. Sci., 54, 1014-1026.

— C. DesAutels, C. Holloway, and R. Korty, 2004: Environmental control of tropical cyclone intensity. J. Atmos. Sci., 61, 843-858.

Frank, W. M., and E. A. Ritchie, 1999: Effects of environmental flow upon tropical cyclone structure. Mon. Wea. Rev., 127, 2044-2061.

— and - 2001: Effects of vertical wind shear on the intensity and structure of numerically simulated hurricanes. Mon. Wea. Rev., 129, 2249-2269.

Garratt, J. R., 1992: The Atmospheric Boundary Layer. Cambridge University Press, 316 pp.

Gray, W. M., 1968: Global view of the origin of tropical disturbances and storms. Mon. Wea. Rev., 96, 669-700.

Grell, G. A., J. Dudhia, and D. R. Stauffer, 1995: A description of the fifth-generation Penn State/NCAR mesoscale model (MM5). NCAR Tech. Note NCAR/TN-398+STR, 122 pp.

Haynes, P., 2002: Turbulence and mixing. Encyclopedia of Atmospheric Sciences, J. R. Holton, Ed., Academic Press, 24462450.

Holton, J. R., 1992: An Introduction to Dynamic Meteorology. 3d ed. Academic Press, 511 pp.

Howard, L. N., and A. S. Gupta, 1962: On the hydrostatic and hydromagnetic stability of swirling flows. J. Fluid Mech., 14, 463-476.

Knaff, J. A., S. A. Seseske, M. DeMaria, and J. L. Demuth, 2004: On the influences of vertical wind shear on symmetric tropi- cal cyclone structure derived from AMSU. Mon. Wea. Rev., 132, 2503-2510.

Kossin, J. P., and M. D. Eastin, 2001: Two distinct regimes in the kinematic and thermodynamic structure of the hurricane eye and eyewall. J. Atmos. Sci., 58, 1079-1090.

— patterns, and rapid pressure falls in hurricane-like vortices. $J$. Atmos. Sci., 58, 2196-2209.

Kwon, Y. C., and W. M. Frank, 2005: Dynamic instabilities of simulated hurricane-like vortices and their impacts on the core structure of hurricanes. Part I: Dry experiments. J. Atmos. Sci., 62, 3955-3973.

McCumber, M., W.-K. Tao, J. Simpson, R. Penc, and S.-T. Soong, 1991: Comparison of ice-phase microphysical parameterization schemes using numerical simulations of tropical convection. J. Appl. Meteor., 30, 985-1004.

McIntyre, M. E., 1989: On the Antarctic ozone hole. J. Atmos. Terr. Phys., 51, 2973-2994.

McWilliams, J. C., 1984: The emergence of isolated coherent vortices in turbulent flow. J. Fluid Mech., 146, 21-43.

_ J. B. Weiss, and I. Yavneh, 1994: Anisotropy and coherent structure in planetary turbulence. Science, 264, 410-413.

Melander, M. V., J. C. McWilliams, and N. J. Zabusky, 1987: Axisymmetrization and vorticity gradient intensification of an isolated two-dimensional vortex through filamentation. $J$. Fluid Mech., 178, 137-159.

Mizuta, R., and S. Yoden, 2001: Chaotic mixing and transport barriers in an idealized stratospheric polar vortex. J. Atmos. Sci., 58, 2616-2629.

Molinari, J., D. Vollaro, and K. L. Corbosiero, 2004: Tropical cyclone formation in a sheared environment: A case study. $J$. Atmos. Sci., 61, 2493-2509.

Montgomery, M. T., and R. J. Kallenbach, 1997: A theory for vortex Rossby-waves and its application to spiral bands and intensity changes in hurricanes. Quart. J. Roy. Meteor. Soc., 123, 435-465.

— , V. A. Vladimirov, and P. V. Denissenko, 2002: An experimental study on hurricane mesovortices. J. Fluid Mech., 471, $1-32$.

- M. M. Bell, S. D. Aberson, and M. L. Black, 2006: Hurricane Isabel (2003): New insights into the physics of intense storms. Part I: Mean vortex structure and maximum intensity estimates. Bull. Amer. Meteor. Soc., 87, 1335-1347.

Nolan, D. S., and M. T. Montgomery, 2002: Nonhydrostatic, three-dimensional perturbations to balanced, hurricane-like vortices. Part I: Linearized formulation, stability, and evolution. J. Atmos. Sci., 59, 2989-3020.

Pagowski, M., and G. W. K. Moore, 2001: A numerical study of an extreme cold-air outbreak over the Labrador Sea: Sea ice, air-sea interaction, and development of polar lows. Mon. Wea. Rev., 129, 47-72.

Persing, J., and M. T. Montgomery, 2003: Hurricane superintensity. J. Atmos. Sci., 60, 2349-2371.

Powell, M. D., P. J. Vickery, and T. A. Reinhold, 2003: Reduced drag coefficient for high wind speeds in tropical cyclones. Nature, 422, 279-283.

Press, W. H., S. A. Teukolsky, W. T. Vetterling, and B. P. Flannery, 1992: Numerical Recipes in Fortran: The Art of Scientific Computing. Vol 1. 2d ed. Cambridge University Press, 933 pp.

Reasor, P. D., M. T. Montgomery, and L. D. Grasso, 2004: A new look at the problem of tropical cyclones in vertical shear flow: Vortex resiliency. J. Atmos. Sci., 61, 3-22. 
Riehl, H., and J. Malkus, 1961: Some aspects of Hurricane Daisy, 1958. Tellus, 13, 181-213.

Robinson, A. R., 1982: Dynamics of ocean currents and circulation: Results of POLYMODE and related investigations. Topics in Ocean Physics, A. R. Osborne and P. MalanotteRizzoli, Eds., North-Holland Publishing, 3-29.

Schecter, D. A., and M. T. Montgomery, 2004: Damping and pumping of a vortex Rossby wave in a monotonic cyclone: Critical layer stirring versus inertia-buoyancy wave emission. Phys. Fluids, 16, 1334-1348.

Schubert, W. H., M. T. Montgomery, R. K. Taft, T. A. Guinn, S. R. Fulton, J. P. Kossin, and J. P. Edwards, 1999: Polygonal eyewalls, asymmetric eye contraction, and potential vorticity mixing in hurricanes. J. Atmos. Sci., 56, 1197-1223.

Simpson, R. H., and H. Riehl, 1958: Mid-tropospheric ventilation as a constraint on hurricane development and maintenance. Proc. Technical Conf. on Hurricanes, Miami, FL, Amer. Meteor. Soc., D4.1-D4.10.

Smith, R. K., M. T. Montgomery, and H. Zhu, 2005: Buoyancy in tropical cyclones and other rapidly rotating atmospheric vortices. Dyn. Atmos. Oceans, 40, 189-208.

Tao, W.-K., and J. Simpson, 1993: The Goddard cumulus en- semble model. Part I: Model description. Terr. Atmos. Oceanic Sci., 4, 35-72.

Vladimirov, V. A., and V. F. Tarasov, 1979: Structure of turbulence near the core of a vortex ring. Sov. Phys. Dokl., 24, 254-256.

_ and - 1980: Elasticity properties of spiral flows. Sov. Phys. Dokl., 25, 526-528.

Wang, Y., 2002: Vortex Rossby waves in a numerically simulated tropical cyclone. Part II: The role in tropical cyclone structure and intensity changes. J. Atmos. Sci., 59, 1239-1262.

Willoughby, H. E., 1998: Tropical cyclone eye thermodynamics. Mon. Wea. Rev., 126, 3053-3067.

Wong, M. L. M., and J. C. L. Chan, 2004: Tropical cyclone intensity in vertical wind shear. J. Atmos. Sci., 61, 1859-1876.

Wu, L., and S. A. Braun, 2004: Effects of environmentally induced asymmetries on hurricane intensity: A numerical study. $J$. Atmos. Sci., 61, 3065-3081.

Zehr, R. M., 2003: Environmental wind shear with Hurricane Bertha (1996). Wea. Forecasting, 18, 345-356.

Zhang, D.-L., Y. Liu, and M. K. Yau, 2002: A multiscale numerical study of Hurricane Andrew (1992). Part V: Inner-core thermodynamics. Mon. Wea. Rev., 130, 2745-2763. 